\title{
Inhibition of return for target discriminations: The effect of repeating discriminated and irrelevant stimulus dimensions
}

\author{
TRACY L. TAYLOR \\ Dalhousie University, Halifax, Nova Scotia, Canada \\ and \\ MICHAEL P. W. DONNELLY \\ Vanderbilt University, Nashville, Tennessee
}

\begin{abstract}
Inhibition of return (IOR) refers to slowed reaction times when a target repeats in the same location as a preceding stimulus. In four experiments, the participants were presented with two successivestimuli, S1 and S2. In Experiments 1 and 2, the participants made a speeded discrimination of the identity or orientation of both S1 and S2 (Experiment 1) or of S2 only (Experiment 2). An IOR effect occurred for the repetition of stimulus location, but a facilitatory effect occurred if the stimulus remained unchanged or if an overt response was repeated. In Experiments 3 and 4, the participants localized S1 and S2 (Experiment 3) or S2 only (Experiment 4) to the left or right of center. In this case, repeating the same stimulus had no effect: IOR occurred any time stimulus location repeated. These results demonstrate that the expression of IOR is modulated by the repetition of a target object, but only when the task requires the discrimination of that object; when no discrimination is required, IOR is unaffected.
\end{abstract}

The capture of attention to the location of an abrupt onset stimulus typically produces an early facilitatory effect for responding to a target that is presented shortly thereafter in the same location (Jonides, 1981; Posner, 1980; Posner \& Cohen, 1984). Even if the initial onset is not spatially predictive, when a target follows at a stimulus onset asynchrony (SOA) of less than approximately $150 \mathrm{msec}$, reaction times (RTs) are facilitated for detecting the target at the previously stimulated location (Posner \& Cohen, 1984). Likewise, RTs are facilitated for localizing a target to the previously stimulated location (Maylor, 1985; Pratt \& Abrams, 1999). The same is true for RTs to report target characteristics, such as identity/form (e.g., Henderson \& Macquistan, 1993) and the direction of a size change (e.g., Klein, 1994). However, when the target follows the initial stimulus by an SOA of more than approximately $300 \mathrm{msec}$, responses become relatively slowed to targets that appear in the previously stimulated location (Posner \& Cohen, 1984) or hemifield (Berlucchi, Chelazzi, \& Tassinari, 2000; Berlucchi, Tassinari, Marzi, \& Di Stefano, 1989; Tassinari, Aglioti, Chelazzi, Marzi, \& Berlucchi, 1987). This later inhibitory

T.L.T. was supported by NSERC; M.P.W.D. was supported by an NIH postdoctoral training grant. Thanks to Jay Pratt and two anonymous reviewers for very helpful comments on an earlier version of this manuscript and to Ray Klein, Jason Ivanoff, and Joe MacInnes for providing constructive feedback on our revisions. Thanks also to Carl Helmick for his assistance in running the Dalhousie participants. Correspondence should be addressed to T. L. Taylor, Department of Psychology, Dalhousie University, Halifax, NS, B3H 4J1 Canada (e-mail: ttaylor2@is.dal.ca). effect is known as inhibition of return (IOR; Posner, Rafal, Choate, \& Vaughan, 1985) and refers specifically to impaired performance that results when a target appears in a previously stimulated location.

The phenomenon of IOR has been the focus of considerable research interest because it represents a possible mechanism for arbitrating between the demands of a changing environment and the need to maintain current goal-directed activity. The initial facilitation of responding that occurs when a target appears in the same location as a preceding onset cue is interpreted as evidence for reflexive orienting to the cue (Jonides, 1981). The visual system is alerted to the environmental variation signaled by the visual transient, and accordingly, attentional resources are deployed to the location of the change. Clearly, if the onset cue predicted the likely location of the impending target, this deployment of attention to the cued location would correspond with the participant's goal of maximizing performance on the target task. However, when the cue is unpredictive with respect to the spatial location of the impending target, the capture of attention to the location of the onset is irrelevant to- or even inconsistent with-the participant's ongoing behavior. As such, reflexive orienting to the distracting stimulus must ultimately be limited in order to prevent further behavioral disruption and possible response perseveration. Hence, the functional significance of IOR is the following: After the initial capture of attention, there is an inhibitory aftereffect that slows responses to stimuli in the cued location and that thereby encourages the search for novelty in visual scanning (Klein, 1988; Klein 
\& MacInnes, 2000; Mueller \& von Muehlenen, 2000; Takeda \& Yagi, 2000).

Initial characterizations of IOR presumed that the inhibition subserved the search for novelty in the visual environment by operating on mechanisms of attention. Posner and Cohen (1984) proposed that slowed responding to targets that appear in a cued rather than an uncued location occurs because attention is inhibited from returning to a location to which it had previously been drawn and subsequently removed. If early facilitation and IOR are both due to attentional mechanisms-attentional orienting on the one hand and the inhibited return of attention on the other-it follows that both should occur together reliably and be measured in comparable ways (see Reuter-Lorenz, Jha, \& Rosenquist, 1996). However, on both issues, the literature is mixed. Although IOR is frequently preceded by early facilitation, the notion that IOR is necessarily preceded by and depends on the initial capture of attention to the location of the peripheral onset cue has not gone unchallenged (Danziger \& Kingstone, 1999; Lambert \& Hockey, 1991; Maylor, 1985; Riggio, Bello, \& Umiltà, 1998; Ro \& Rafal, 1999; Tassinari, Aglioti, Chelazzi, Peru, \& Berlucchi, 1994; although see Berger, Dori, \& Henik, 1999). Indeed, it is not even clear that IOR requires peripheral events; IOR has been reported in conditions for which nonpredictive central arrows are used to generate IOR (Taylor \& Klein, 2000). Similarly, the issue of whether facilitation and IOR are both sensitive to the same stimulus-response parameters has been contentious. This is especially true where the nature of the target task is concerned.

As was described above, early facilitation following peripheral cues occurs for target detection and localization, as well as for a variety of target discriminations. However, this is not universally true of IOR. Although there are reports that IOR can be obtained for the discrimination of target identity and orientation (Danziger, Kingstone, \& Snyder, 1998; Handy, Jha, \& Mangun, 1999; Lupiáñez, Milán, Tornay, Madrid, \& Tudela, 1997; Pratt, 1995; Pratt \& Abrams, 1999; Pratt, Kingstone, \& Khoe, 1997), there are also documented failures to obtain IOR for the discrimination of target color, spatial displacement (vernier offset), orientation, luminance, and identity (Kwak \& Egeth, 1992; Tanaka \& Shimojo, 1996; Terry, Valdes, \& Neill, 1994).

Although a purpose of our investigation is to clarify the conditions under which IOR is and is not observed for target discriminations, at this juncture suffice it to say that failures to show a consistent IOR effect for such tasks brought the attentional hypothesis under close scrutiny. In two separate reviews, Klein and Taylor (1994) and Taylor and Klein (1998) reinterpreted the extant IOR literature in terms of an alternative, motor, view. Whereas the prevailing attentional account presumed that slowed reorienting of attention led to slowed perceptual processing of targets presented at cued locations, the motor view argued that IOR resulted from a bias against responding to targets that appeared in the same location as a preceding cue. In other words, IOR reflected a reluctance to respond to targets in a previously cued location, and not the inhibited return of attention to that location.

Although the motor bias view of IOR accommodated a large body of literature, it did not wholly supplant an attentional view. As is often the case with "either-or" debates, it has become apparent that IOR likely does not reflect the operation of a single mechanism. Indeed, as testimony to our growing appreciation of the complexities of IOR, there is evidence that IOR in the visual modality (1) affects perceptual sensitivity in signal detection (Handy et al., 1999; although see Taylor \& Klein, 1998), (2) operates differentially on low- and highfrequency words in lexical decision tasks (Chasteen \& Pratt, 1999), (3) interacts with semantic inhibition (Fuentes, Vivas, \& Humphreys, 1999a), (4) results in P1 and $\mathrm{P} 2$ component reductions in event-related potentials (McDonald, Ward, \& Kiehl, 1999), (5) reverses semantic priming and flanker effects (Fuentes, Vivas, \& Humphreys, 1999b), (6) is mediated by attentional control settings (Gibson \& Amelio, 2000; Klein, 2000) and can be modulated by endogenous attention (Berger \& Henik, 2000; although see Berlucchi et al., 2000), (7) may be coded in different reference frames, depending on the nature of the stimuli and response modality (Abrams \& Pratt, 2000; Becker \& Egeth, 2000; Howard, Lupiáñez, \& Tipper, 1999; Maylor, 1985; Tipper, Jordan, \& Weaver, 1999), (8) can be tagged to both locations and to static or dynamic objects (Gibson \& Egeth, 1994; Jordan \& Tipper, 1998, 1999; Ro \& Rafal, 1999; Tipper, Driver, \& Weaver, 1991; Tipper et al., 1999; Tipper, Weaver, Jerreat, \& Burak, 1994), (9) can be tagged to multiple spatial locations (Danziger, Kingstone, \& Snyder, 1998; Snyder \& Kingstone, 2000), and (10) reflects a complex interplay between perceptual/attentional and motor influences (Abrams \& Dobkin, 1995; Kingstone \& Pratt, 1999; Rafal, Egly, \& Rhodes, 1994; Taylor \& Klein, 2000). In light of this multifaceted nature of IOR, it behooves us to understand the boundary conditions under which inhibition is and is not observed; a comprehensive integrative model of the mechanism and function of IOR will be impossible otherwise.

In an attempt to better understand the boundary conditions for obtaining IOR in visual tasks, we are interested in reevaluating the role that IOR may play in target discriminations. This is because the issue of when and whether IOR occurs for target discriminations has proven to be central to theorizing about the mechanisms of inhibition. Yet, as we hope to make clear, there are some critical discrepancies in the literature that need to be resolved before IOR can be adequately characterized. As a first step in outlining these discrepancies and our consequent purpose and methods, it is necessary to distinguish between the various paradigms that have been used to study IOR and between the types of responses that can be made to report a target discrimination. 


\section{Paradigms Used to Study IOR}

There are four paradigms that have typically been used to explore IOR. In a cue-target paradigm, two stimuli-a cue and a target-are presented on each trial. The cue and the target are physically distinct (usually, the cue is the brightening of a peripheral stimulus box, but it may also be the onset of a nontarget stimulus in a peripheral location), and participants are required to respond only to the target. IOR is measured as relatively slower RTs when the target repeats after a relatively long SOA in the same location as the cue. In a continuous-responding paradigm, only one stimulus - a target-is presented on each trial. Participants are required to respond to each target, and IOR is measured as relatively slowed RTs when the target on trial $N$ repeats, after a relatively long response-stimulus interval (RSI), in the same location as the target on trial $N-1$. In a target-target paradigm, which is conceptually similar to the continuous-responding paradigm, two targets separated by a fixed SOA are presented in succession on a discrete trial. The participant is required to respond to both targets, and IOR is revealed as slower responding to the second target when it appears in the same location as the first target. Finally, in a no-response-target paradigm, the trial structure is identical to a target-target paradigm, except that a response is required only to the second of the two targets. Although the first stimulus essentially serves as a cue, a no-response-target paradigm is distinguished from a cue-target paradigm in that both stimuli presented on a trial are drawn from a target pool; in a cue-target paradigm, the stimuli are visually distinct, and the cue does not map onto any response (either executed or withheld).

\section{IOR for Target Discriminations}

As the term has generally been applied in the literature, discrimination is reserved to describe tasks that require a perceptual analysis of the features that make up a target object. Although the term location discrimination has occasionally been used to refer to tasks in which the participant must indicate the location of an onset target, this is more accurately considered a choice localization response. Under the terminology that we will apply throughout the following, a discrimination task does not necessarily presume a choice response, nor does a choice response necessarily imply that a discrimination has been performed. Instead, a target task is conceptualized as a discrimination when it requires the participant to distinguish more than the simple occurrence or location of any onset. If, for example, a participant were required to detect the onset of any stimulus in the visual field or to localize any onset, regardless of its physical features or identity, this would not be classified as a discrimination. However, if the participant were required to process characteristics of the target stimulus (other than its onset or location) before being able to execute an appropriate response (simple or choice), this would be considered a target discrimination. Examples of discriminations in- clude distinguishing target color, identity, shape, orientation, and so forth.

Regardless of the paradigm used, the study of IOR in target discrimination tasks can be categorized further according to the relationship between the discrimination that is made and the response used to report the result of that discrimination. In one class of tasks, the discrimination criteria are used to determine whether an onset is the target or not; the executed response is intended to confirm the presence of the target by signaling either its onset or its location. For the sake of brevity, we will refer to these tasks as occurrence discriminations.

As an example of an occurrence discrimination, imagine that a participant is presented with a single stimulus on each trial and is asked to press a button when the stimulus is red but to withhold a response if the stimulus is blue. With the target thus defined as a red stimulus, this go/no-go task requires only that the participant determine whether any given onset stimulus is red or not. This binary decision is then reflected in the participant's decision to make a response (yes, the stimulus is red) or not (no, the stimulus is not red). As such, the task can essentially be conceived of as the determination of whether or not the target has occurred.

In a go/no-go task, it is necessary to present trials on which no target is presented. This limits the amount of data that is available to measure any IOR effects that might be present. A solution to this problem is to present a localization task in which a discrimination target is presented simultaneously with a distractor. Going back to our example, a red target would appear on every trial; however, it would appear simultaneously with the blue distractor. To ensure that the color criterion was being applied and red per se was being detected, the participant would be asked to indicate the location where the red target occurred.

In the other class of tasks, the discrimination criteria are applied to a target to determine which task-relevant characteristics are present in the object; the executed response reports the state of the object with respect to the discriminated dimension. An example of such a task would be a red/blue discrimination in which a single target is presented on each trial. Rather than predefining the target that participants would be required to detect or localize (e.g., red or blue), both colors would represent alternate target states. That is, equal numbers of red and blue targets would be presented, and the participant's task would be to identify the color of the target on each trial. One unique overt response would indicate that a red target had been presented; a different response would indicate that a blue target had been presented. These tasks report the state of the discriminated object characteristic (e.g., color, orientation, shape) regardless of location; for the sake of brevity, we will refer to these tasks as object discriminations.

The distinction between occurrence and object discriminations is an important one. To the extent that IOR 
may subserve effective visual search, it should aid decisions about what an object is, in addition to decisions about whether a particular object is present and where it is located. In real life, our visual searches are seldom limited to the determination of whether a discriminated target has occurred or not; more often, we are required to identify a searched-for item as a member of a target class. For example, rather than simply determining whether an illuminated traffic control signal is red or not, we seek to determine whether it is red, yellow, or green. As such, it is important to know whether IOR is useful only for the search for a predefined target (e.g., "is the illuminated signal red?") or whether it may also affect the search to identify a member of the target class (e.g., "what is the color of the illuminated signal?"). In the following, evidence of IOR for occurrence and object discriminations will be examined in turn.

\section{Occurrence Discriminations}

As has been stated, occurrence discriminations report the presence of a discriminated target by signaling either its appearance or its location. Table 1 outlines stimulus and response conditions that have been used to examine IOR for occurrence discriminations. As can be seen, this class of discrimination has been used across a range of paradigms and using a variety of responses. In the following, we will highlight three studies that have required simple detection responses, manual go/no-go decisions, and target localizations, respectively, to signal the occurrence of discriminated targets in a cue-target paradigm.

As a first example, consider a study performed by Danziger et al. (1998), in which a discrimination of stimulus identity was used to determine the onset of a target in a multiple cue-target paradigm. Danziger et al. presented participants with an array of circular placeholders that marked the locations at which cues and targets could appear. Cues consisted of the changing of one of the circles to a "Z"; targets consisted of the changing of one of the circles to an "N." On a given trial, one, two, or three cues could be presented sequentially. With each change in one of the circular placeholders, participants were required to analyze the visual form to determine whether the onset was a cue or a target identity. When a change was identified as a " $\mathrm{Z}$ " (rather than as an "N"), the participant was required to make a simple buttonpress response to report the presence of this target. IOR was observed at the most recently cued location, as well as at locations up to three cues back in time.

The sequential presentation of multiple cues forced a target discrimination in Danziger et al.'s (1998) study and thereby allowed the use of a simple detection response. In a more typical IOR paradigm, inhibition is measured relative to the location of only one preceding stimulus (whether a cue or a target). In this situation, it is impossible to use a simple detection response to report a target discrimination: Pressing a button upon the onset of a discrimination target would not communicate any information other than an awareness of the onset itself; there would be no indication that the required discrimination was actually performed. This is where a go/no-go procedure has been used instead. In just such a task, Handy et al. (1999) presented participants with a cue that was followed, after a long delay, by a horizontally or vertically oriented line target. In this go/no-go task, participants had to make a simple buttonpress response to report the appearance of the target (horizontal for half of the participants, vertical for the other half) and to withhold a response in the absence of a target. As with the simple detection response of Danziger et al., IOR was observed for this go/no-go occurrence discrimination.

Similarly, Pratt et al. (1997) demonstrated IOR in a cue-target paradigm that required a spatial localization. They presented participants with an onset cue to the left or right of fixation. This was followed, after a relatively long delay, by two stimuli-an "X" and a "+"—one in each of two peripheral stimulus boxes. One of the stimuli was defined as the target; the other was defined as the distractor. The participant's task was to make a speeded buttonpress response to report the location (left or right) of the target, which was an " $\mathrm{X}$ " for half of the participants and a "+" for the other half. RTs to localize the target were slower when it occurred in the same versus a different location as the cue-the hallmark of IOR. Similar findings have been observed when saccadic eye movements have been used to localize the discriminated target (Pratt, 1995).

Although the preceding examples all used cue-target paradigms, IOR for occurrence discriminations has also been examined by using continuous-responding, targettarget, and no-response-target paradigms. As can be seen in Table 1, the majority of studies have revealed IOR. However, there are a few exceptions. The most notable exception is that IOR does not occur reliably in targettarget tasks that require a saccadic localization to the first discrimination target (Pratt \& Abrams, 1999). Indeed, in order to observe IOR for a saccadic-manual response combination, it is necessary for the first and the second targets to be drawn from different stimulus pools, or in the case of letter stimuli, it is necessary to limit the exposure duration of the first stimulus. The ability to find IOR when the first response is a manual localization is not restricted in the same way; the reason for this discrepancy between first-target responses is not clear. ${ }^{1}$

The only other exception comes from a study by Terry et al. (1994) that reported no IOR for continuousresponding paradigms that utilized either manual go/nogo or manual localization responses. It is possible that IOR simply cannot be obtained with such a paradigm; however, it is more likely that Terry et al.'s failure to observe IOR was due to repetition priming from their letter stimuli. In both tasks (go/no-go, localization), participants had to detect or localize one of two possible letter 


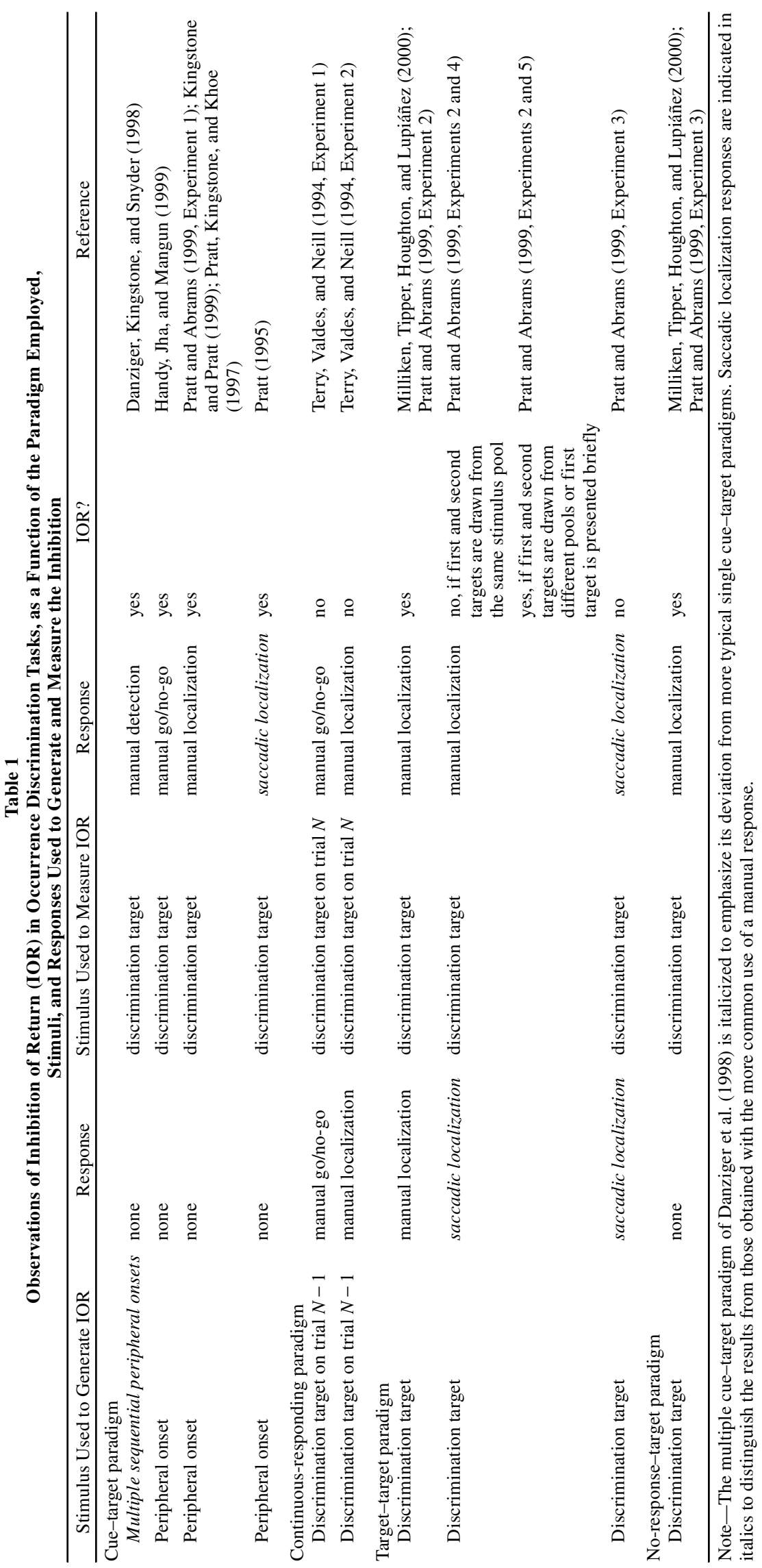


targets (A/B). RTs were faster when the same target letter was presented in the same location than when the same target letter appeared in the opposite location. However, RTs were slower when a different target letter was presented in the same location than when the different target letter appeared in the opposite location. Although this latter effect was only $9 \mathrm{msec}$ for the go/nogo discrimination and $15 \mathrm{msec}$ for the localization task, it is consistent with the suggestion that location-based IOR may have otherwise appeared in their task, except that it was overridden by a priming effect owing to the repetition of the same letter. Indeed, when Pratt and Abrams (1999) required participants to discriminate target shapes rather than letters, they reported that IOR does occur for manual-manual localization responses made within a target-target paradigm (which is conceptually similar to the continuous-responding paradigm used by Terry et al., 1994).

These exceptions notwithstanding, it is clear that IOR for occurrence discriminations is observed across a variety of paradigms. On the surface, this would suggest that IOR for perceptual discriminations is a robust phenomenon. However, on the basis of this evidence, it is not clear whether the inhibition is for the discrimination itself or for the response needed to signal the result of that discrimination. In other words, does IOR operate to slow the perceptual processing of the discriminated target? Or does IOR operate to slow responses that are made, either implicitly or explicitly, toward information presented at the inhibited location? Consider the following.

To the extent that covert attention facilitates the processing of signals presented at a cued location (e.g., Downing, 1988; Hawkins et al., 1990; Mangun \& Hillyard, 1991), the view that IOR represents an inhibitory effect on attention predicts slowed perceptual processing at the previously cued location. That is, the speed to discriminate a target presented at the previously stimulated location should be slowed, and in turn, this slowed perceptual processing should be manifest in slowed RTs to report the appearance or location of the discriminated target. Confirming this prediction with respect to occurrence discriminations, however, is uninformative with respect to the issue of whether IOR has an attentional basis. This is because the motor hypothesis likewise predicts that occurrence discriminations should be subject to IOR.

Recall that to report the occurrence of a discriminated target, participants make either a detection response, for which they press a single button to indicate that a predefined target has appeared, or a localization response, for which they make an eye movement or press a button to indicate the location of the discriminated target. The motor view states that IOR represents a reluctance to respond to information presented at the inhibited location (Klein \& Taylor, 1994; Taylor \& Klein, 1998). This would impact both eye movements and choice localization responses. Moreover, the motor hypothesis explic- itly states that detection (including go/no-go) responses would also be affected, under the "assumption that, normally, detection responses are implicitly 'to a spatial location.' In essence, there is a criterion shift for responding that something has happened at a particular location" (Klein \& Taylor, 1994, p. 143).

Even without adopting an attention-or-motor stance, given the key role that IOR is thought to play in visual search, it is important to distinguish between effects that may operate on attentional mechanisms and those that may operate on motor responses. This is particularly true, given that attentional and motor effects have been shown to depend on the particular stimulus-response combination used to assess IOR (Taylor \& Klein, 2000). Because IOR for occurrence discriminations cannot distinguish between attentional and motor effects, determining the role that IOR might play in slowing perceptual processing requires an evaluation of object discrimination tasks. ${ }^{2}$

\section{Object Discriminations}

A response that reports the result of a perceptual discrimination, rather than simply the presence or location of a discriminated target, represents an object discrimination task. The stable mapping between the discriminated target dimension and the response takes the form of a choice task in which a unique buttonpress maps onto each target state. Table 2 presents studies that have examined IOR effects for object discriminations. As is immediately obvious upon inspection of this table, there have been a limited number of paradigms employed for this type of discrimination, and those that have been employed have produced mixed results.

As is shown in Table 2, IOR can be reliably produced in cue-target paradigms that use manual responses to report the outcome of the discrimination-so long as the cue is an onset and can be processed with the same attentional control settings as those used to process the target. This was demonstrated by Gibson and Amelio (2000). They presented participants with a cue to one of four peripheral stimulus boxes. On any given trial, the cue could be a brightening of one of the stimulus boxes (onset cue) or else the changing of one of the stimulus boxes to red (color cue). This was followed after a variable SOA by a target. In an onset target condition, the target was a "U" or an " $H$ " that was onset suddenly in one of the four possible target locations. In a color target condition, the target was a red "U" or " $\mathrm{H}$ " that appeared along with distractors at the four nontarget locations. Regardless of the target condition, participants were required to determine the identity of the target and to register this response by pressing one key for " $U$ " and another for "H." Consistent with the notion of an attentional set (see Folk, Remington, \& Johnston, 1992), onset cues resulted in IOR for onset targets, but not for color targets. However, the obverse was not true; IOR never occurred following color cues. Thus, at least when 


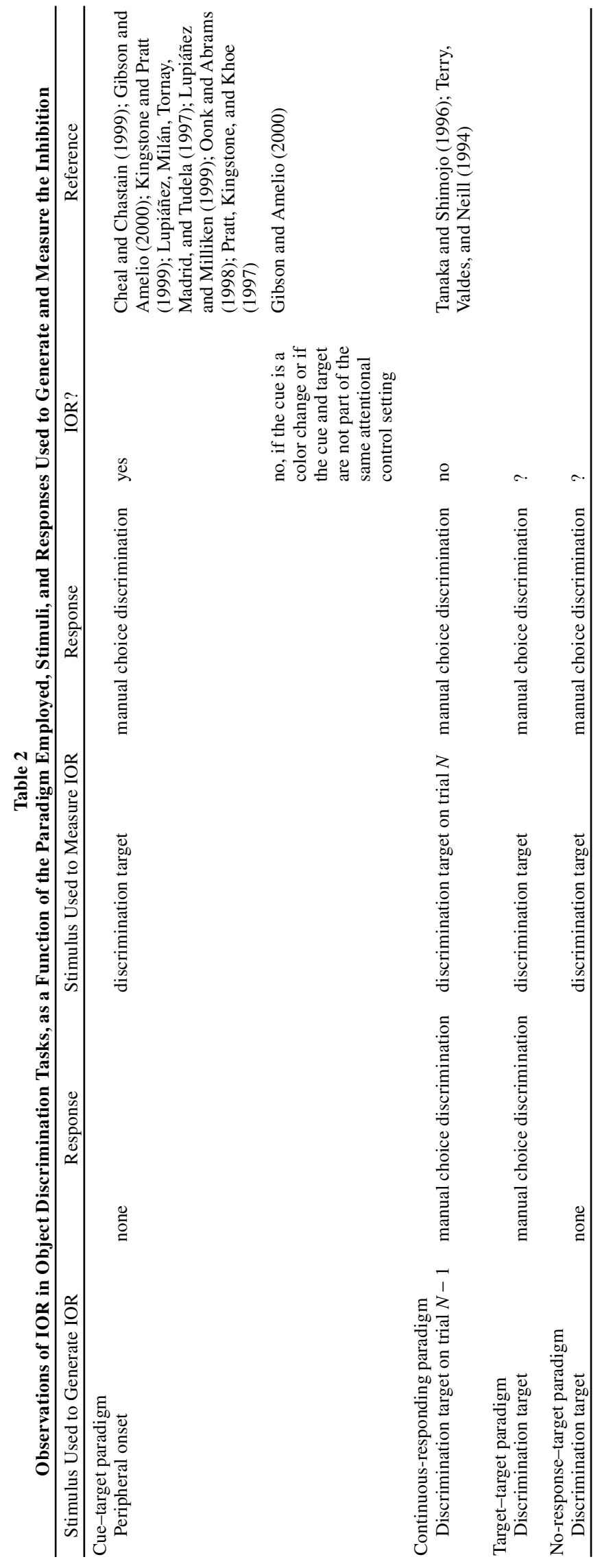


the cue and the target require compatible attentional control settings, IOR can be obtained for object discriminations made within a cue-target paradigm (see also Cheal \& Chastain, 1999; Lupiáñez et al., 1997; Oonk \& Abrams, 1998; Pratt et al., 1997).

This effect does not generalize to a continuousresponding paradigm. Tanaka and Shimojo (1996) presented participants with colored rectangles that were oriented horizontally or vertically and that appeared to the upper right or upper left of fixation. In object discrimination conditions (our term, not theirs), participants were presented with one target on each trial and were required to make a speeded buttonpress to report its color (red/green) or orientation (vertical/horizontal). IOR was not observed for these responses, nor was IOR observed for similar object discriminations of target size, spatial displacement (vernier offset), luminance, and shape.

With respect to object discrimination tasks, it is unclear what factors contribute to the different outcomes obtained with cue-target and continuous-responding paradigms. However, at least four possibilities present themselves. First, given the relatively longer RTs associated with making object discrimination responses versus detection/localization responses (e.g., Tanaka \& Shimojo, 1996), the use of RSI in the continuous-responding procedure might result in a failure to sample IOR within the SOA range in which the inhibition is most likely to be present. However, in light of the fact that IOR for discriminating a target has been shown to follow a longer time course than IOR for detecting a target (Lupiáñez et al., 1997), this seems unlikely.

A second reason why IOR may occur in cue-target paradigms, but not in continuous-responding paradigms, could be an inability of an object discrimination to generate inhibition in the first place and/or to reveal its effects when present (see Taylor \& Klein, 1998). That is, in a continuous-responding paradigm, the target on trial $N$ measures inhibitiongenerated by the target on trial $N-1$ and generates inhibition for the target on trial $N+1$. One way to avoid these sequential dependencies is to utilize a target-target paradigm. Like the continuous-responding paradigm, participants respond to all targets in a targettarget paradigm. However, by presenting pairs of targets in discrete trials, the first target is used only to generate IOR, and the second is used only to measure its effects (any potential influence from the second target on one trial to the first target on the subsequent trial is minimized by imposing relatively long intertrial intervals). If the dual generate-and-measure role played by targets in the continuous-responding paradigm is responsible for the lack of IOR for object discriminations, IOR will occur for this task when the paradigm is changed to one that uses a target-target procedure. If, however, the dual role is not the sole reason for the finding of no significant IOR, presenting sequential targets in a target-target procedure will have no effect on this outcome. One goal of the present experiment is to test these predictions by pre- senting participants with an object discrimination task in the context of a target-target paradigm.

A third reason why IOR may occur for object discriminations in a cue-target paradigm, but not in a continuousresponding paradigm, is that the usual requirement to respond to every stimulus in the latter interferes specifically with IOR for object discriminations. If this is the case, IOR will not be observed for object discriminations made within a target-target task either. However, if a noresponse-target task is used, according to this account, IOR should be observed just as it is for cue-target paradigms. In the present investigation, this possibility will also be explored.

A final reason for the discrepancy between cue-target and continuous-responding paradigms with respect to object discriminations is that there may be interactions among repeated features of the discriminated target that interfere with location-based inhibitory tagging. When making an object discrimination in a cue-target paradigm, location is the only salient stimulus dimension that typically repeats. This contrasts with the continuousresponding paradigm, for which attributes other than target location can also repeat. To demonstrate the differences between cue-target and continuous-responding paradigms with respect to repeated stimulus dimensions, consider a task that requires participants to discriminate the shape of a target (a vertically oriented rectangle or ellipse) that appears to the left or right of central fixation. A speeded buttonpress with the index finger reports a rectangle; a speeded buttonpress with the middle finger reports an ellipse. In a cue-target paradigm, the target is preceded by a cue that requires no overt response. This cue does not have the same visual form as the target and, instead, is an abrupt onset of a different form-for example, the brightening of a stimulus box-in the periphery. Thus, from cue to target, there is no repetition of overt response and no repetition of visual form identity. Location is the only stimulus characteristic that can repeat. Contrast this with a continuous-responding paradigm. To use the extreme example of a vertically oriented rectangle presented to the left on both trials $N-1$ and $N$, it becomes clear that up to four dimensions may repeat: (1) visual form identity (rectangle), (2) stimulus orientation (vertical), (3) overt response (a buttonpress with the index finger to report a rectangle), and (4) location of the stimulus (to the left of fixation).

Supporting the notion that the repetition of object characteristics may be key to understanding why IOR does not occur for object discriminations in continuousresponding paradigms, facilitatory, rather than inhibitory, effects have been observed when the same target is repeated in the same rather than in a different location (e.g., Tanaka \& Shimojo, 1996; Terry et al., 1994). However, it is not clear whether the reported facilitation was due to the repetition of an overt response, the repetition of the target stimulus, or perhaps, to some combination of the two. Furthermore, in these studies, 
the data were collapsed to reveal RT effects when the discriminated target feature changed or repeated, with little or no clear indication of whether IOR occurred when a nondiscriminated feature of the target changed versus repeated. A primary goal of the present research is to examine IOR as a function of whether a target repeats a discriminated feature, a nondiscriminated feature, or both in the context of an object discrimination.

In a series of four experiments, we examined the effects of repeating stimulus location when no stimulus dimensions repeated or when identity, orientation, location, and response repeated alone or in combination. As was alluded to previously, two stimuli, referred to as S1 and S2, respectively, were presented to the left or right of fixation on each trial. S2 was presented at a fixed SOA of $1,000 \mathrm{msec}$ relative to $\mathrm{S} 1$, an interval shown to produce robust IOR effects whether a response is required to one or to both stimuli (Taylor \& Klein, 2000). The participants were required to respond to both stimuli (target-target paradigm) or else, only to the second stimulus (noresponse-target paradigm). In Experiments 1 and 2, an object discrimination task was employed; in Experiments 3 and 4 , the effects of repeating versus changing a target object were examined in a task that did not require target discrimination.

It may be the case that reports in the literature are accurate and that IOR for object discriminations occurs only in cue-target paradigms or when no more than one target is presented in succession. If so, we would be forced to conclude that IOR for object discriminations is not robust across large changes in experimental paradigm and that its utility in subserving the search for novelty is therefore seriously limited by task demands. Alternatively, it may be that there is an inhibitory effect for repeating stimulus location that is modulated by interactions with other repeated stimulus dimensions and/or responses (see Tanaka \& Shimojo, 1996; Terry et al., 1994). It has typically been an unstated assumption that any unpredictive onset event in the periphery, regardless of its visual features, is sufficient to generate IOR. To the extent that there may be interactions between the features of the stimulus used to generate the inhibition and the features of the stimulus used to measure the consequences of the inhibition, our models of IOR will be forced to take into account the relationship between these two visual events (see Klein, 2000; Taylor \& Klein, 2000). And given that there is already uncertainty about whether IOR occurs reliably for object discriminations, determining the stimulus/response parameters under which IOR does and does not occur is critical to our understanding of the mechanisms underlying this inhibitory effect.

\section{EXPERIMENT 1}

In Experiment 1, S1 and S2 were drawn independently from a pool of four possible targets that could appear in one of two possible locations. Responses required an ob- ject discrimination of target orientation or identity, so that two of the targets mapped onto a buttonpress with the index finger and the other two mapped onto a buttonpress with the middle finger. In Experiment 1, the participants responded to both S1 and S2. The overall design of Experiment 1 resulted in the combined repetition of the stimulus (1) orientation, identity, and location, (2) orientation and identity, (3) orientation and location, (4) orientation, (5) identity and location, (6) identity, (7) location, or (8) none. As well, depending on the discriminated dimension (identity or orientation), response could also repeat from S1 to S2.

From these repetition conditions, it was possible to derive measures of IOR as a function of whether location repeated alone or in the context of other stimulus/response dimensions. The difference between (1) and (2) above provided a measure of IOR in the context of repeated orientation and identity; the difference between (3) and (4) provided a measure of IOR in the context of repeated orientation; the difference between (5) and (6) provided a measure of IOR in the context of repeated identity; and the difference between (7) and (8) provided a "pure" measure of IOR - the effect of repeating location when no other stimulus dimensions repeated. More will be said about these contextual IOR effects in the Method section.

\section{Method}

Participants. Eighteen undergraduate students from Vanderbilt University and 10 undergraduate students from Dalhousie University volunteered to participate in exchange for psychology course credit. The participants were tested either individually or in pairs in a single experimental session that required approximately $1 \mathrm{~h}$ for the Vanderbilt students and $0.5 \mathrm{~h}$ for the Dalhousie students. All the participants reported normal or corrected-to-norm al vision and were naive as to the experimental purpose.

Stimuli and Apparatus. Three outline boxes were aligned horizontally across the center of an Apple Color Plus 14-in. (Vanderbilt) or a ViewSonic 17-in. PT775 (Dalhousie) color display monitor. When viewed at a distance of $57 \mathrm{~cm}$, the outline boxes were $1.5^{\circ}$ of visual angle on a side and separated by $3.9^{\circ}$ of visual angle center to center. The fixation stimulus was a cross consisting of two intersecting $0.8^{\circ}$ lines presented in the center of the middle outline box. The two stimuli (S1 and S2) presented on each trial were filled rectangles or ellipses that were oriented horizontally or vertically and presented in the boxes to the left or right of the center box. Rectangles and ellipses were both $1.1^{\circ}$ across the long axis and $0.7^{\circ}$ across the short axis. All stimuli were yellow presented on a uniform black background. Stimulus presentation and data collection were controlled by a 7100/80 Macintosh PowerPC (Vanderbilt) or a Macintosh G4-400 (Dalhousie) microcomputer running customdesigned experimental software (written in C) that incorporated accepted routines for synchronizing stimulus presentation with the monitor raster cycle. The participants responded on an AppleDesign extended keyboard (Vanderbilt) or a Macintosh G4 universal serial bus keyboard (Dalhousie).

Procedure. The participants placed the index and middle fingers of their right hands on the " 1 " and " 2 " keys of the numerical keypad, respectively; the mouse button was placed near the left hand. The participants were instructed to click the mouse button when they were ready to initiate a trial and to try to maintain fixation throughout the trial duration. Because inhibition occurs 


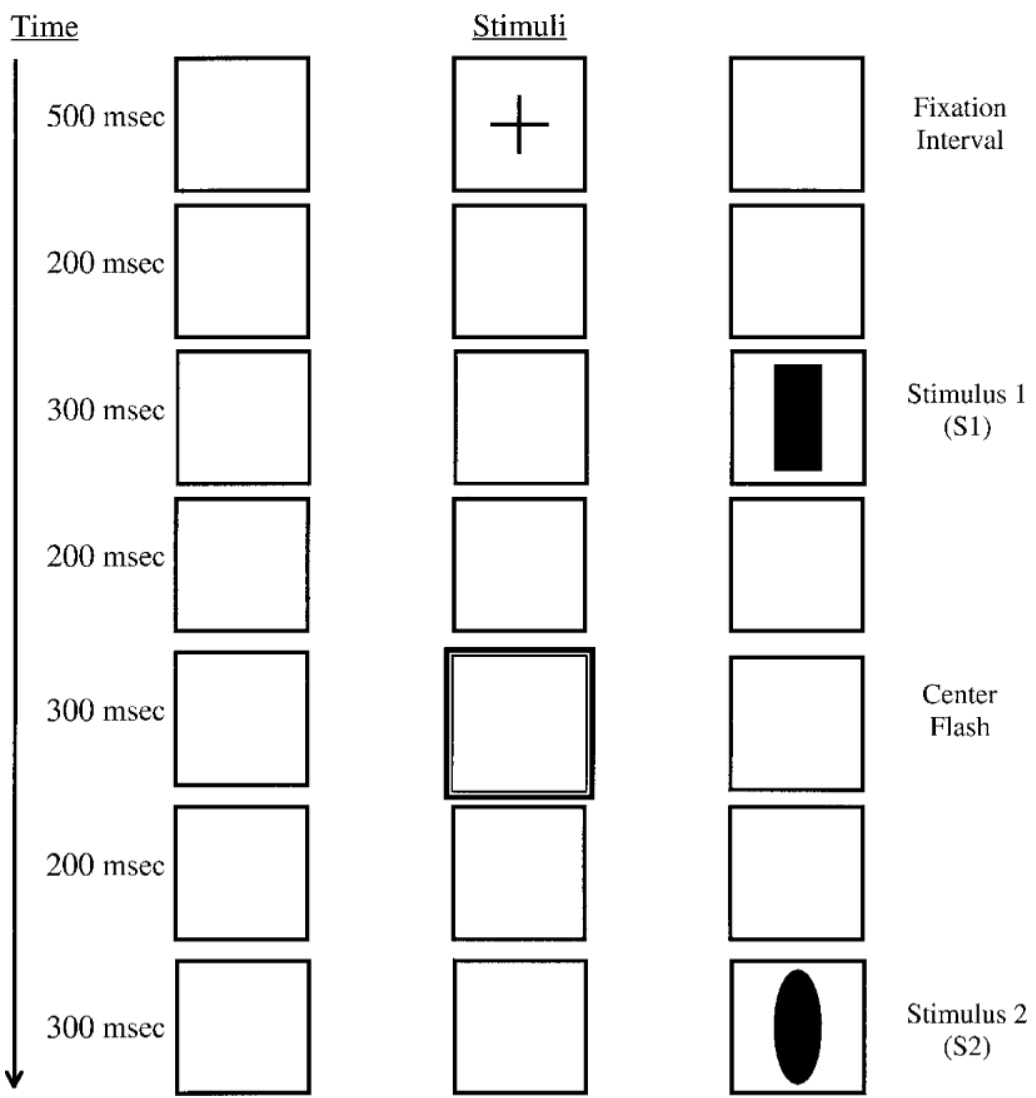

Figure 1. Method used in Experiments 1 and 2.

whether or not saccades are made to the initial onset stimulus (Rafal, Calabresi, Brennan, \& Sciolto, 1989; Taylor \& Klein, 2000), to the second stimulus (Abrams \& Dobkin, 1995; Reuter-Lorenz et al., 1996; Taylor \& Klein, 2000), or to both (Rafal et al., 1994; Taylor \& Klein, 2000; Vaughan, 1984), eye movements were not monitored.

Trial events are depicted in Figure 1. Following trial initiation, the fixation cross was presented for $500 \mathrm{msec}$. Then, the first stimulus (S1) was presented for $300 \mathrm{msec}$. On a pseudo-random half of the trials, S1 appeared in the box to the left of fixation; on the other half of the trials, S1 appeared in the box to the right of fixation. S1 was a horizontally or vertically oriented ellipse or rectangle. Half of the participants from each university (Vanderbilt and Dalhousie) performed in the identity discrimination condition: They pressed the " 1 " on the numerical keypad to report a vertically or horizontally oriented rectangle and the " 2 " to report a vertically or horizontally oriented ellipse. The other half of the participants from each university performed in the orientation discrimination condition: They pressed " 1 " to report a vertically oriented rectangle or ellipse and "2" to report a horizontally oriented rectangle or ellipse. If the participants failed to respond to $\mathrm{S} 1$ between 80 and $500 \mathrm{msec}$ after its presentation, or else if they responded incorrectly, the trial was aborted and recycled later in the block. The participants were given $833 \mathrm{msec}$ of visual feedback to indicate the nature of their error- "Too Soon" if they responded in less than $80 \mathrm{msec}$, "Error" if they pressed the incorrect response key, and "Miss" if they failed to respond or had a latency greater than $500 \mathrm{msec}$. The screen was cleared, and the error message (printed in blue, 12-point Chicago font) was centered in an otherwise black field. If the participants responded to S1 correctly and within the time limits, no feedback was given, and the trial proceeded.

In the absence of an error, after a further $200 \mathrm{msec}$ (i.e., at a 500msec SOA, relative to $\mathrm{S} 1$ ), the box at fixation brightened for $300 \mathrm{msec}$. This brightening was accomplished by plotting a second yellow box 1 pixel outside the perimeter of the original fixation box. If the participants pressed any key during the central brightening, the screen cleared, and the error message "Do Not Respond to Central Flash" appeared; the trial was aborted and later recycled. Otherwise, after $200 \mathrm{msec}$ (i.e., at a 500-msec SOA, relative to fixation brightening), S2 was presented for $300 \mathrm{msec}$.

As with S1, S2 was one of the four stimuli presented pseudorandomly with equal probability to the left or right of the central box. The participants in the identity discrimination condition made a speeded buttonpress to report the identity of $S 2$; the participants in the orientation discrimination condition made a speeded buttonpress to report the orientation of $\mathrm{S} 2$. The participants were informed that there was no relationship between the S1 and S2 stimuli, and they were instructed to respond as quickly and as accurately as possible.

If the participants failed to respond to $\mathrm{S} 2$ between 80 and $1,000 \mathrm{msec}$ after its presentation, or else if they responded incorrectly, they were given $833 \mathrm{msec}$ of visual feedback (as described for $\mathrm{S} 1$ ) to indicate the nature of their error. If the participants responded correctly and within the time limits, they were also informed by a message that read "Correct." Trials that had anticipations $(\mathrm{RT}<80 \mathrm{msec}$ ), misses (no response or RT $>1,000 \mathrm{msec}$ ), 
or incorrect responses were not recycled. The response to S2 was followed by a $1,500-\mathrm{msec}$ forced intertrial interval. After this interval, the participants were free to initiate the next trial at will.

Design. The stimulus pool from which S1 and S2 were independently drawn will be designated as follows: vertical rectangle (VR), horizontal rectangle (HR), vertical ellipse (VE), and horizontal ellipse (HE). Each of these four S1s was presented equally often and with equal probability to the left and right of fixation. Likewise, each S2 was presented equally often and with equal probability to the left and right of fixation. For each of the $\mathrm{S} 1$ stimuli presented at each of the two locations (left, right), this resulted in eight relationships of S2 to S1 (repetition conditions). To indicate the repetition of a stimulus dimension from S1 to S2, we will use the first letter of the repeated characteristic to indicate that this was the case; if one of these stimulus characteristics did not repeat from S1 to S2, we will use a placeholder, a tilde ( ), to indicate which dimension did not repeat. When describing the repetition conditions, we will indicate the repetition of orientation $(O)$, identity $(I)$, and location $(L)$, in that order. To capture the fact that participants responded to both $\mathrm{S} 1$ and $\mathrm{S} 2$, the repetition condition labels will also be appended with information about whether a response $(R)$ repeated. Although this information is redundant with information about whether the discriminated stimulus dimension (identity or orientation) repeated, it is important to emphasize that any repetition effects may be due to the repeated discriminated dimension, the repeated response, or both.

To demonstrate the repetition conditions in an identity discrimination task, Figure 2 shows a single $\mathrm{S} 1$ presentation and all the possible S2 presentations. Using the key designations adopted for the present study, this example shows a press of the "1" key to report a rectangle and a press of the " 2 " key to report an ellipse. The $S 1$ is a VR presented to the right. This $S 1$ would be followed equally often by each of four different S2 identities presented to the left or right of fixation (these are represented under the label "S2 Presentation"). As is shown, S2 could repeat the same orientation (vertical, $V$ ), identity (rectangle, $R$ ), location (right, $R$ ), and response (" 1 ") as $\mathrm{S} 1$. This resulted in eight repetition conditions wherein, relative to S1, S2 could repeat: OILR (orientation, identity, location, and response); $O I \sim R$ (orientation, identity, and response); $O \sim L \sim$ (orientation and location); $O \sim \sim \sim$ (orientation); $\sim I L R$ (identity, location, and response); $\sim I \sim R$ (identity and response); $\sim \sim L \sim$ (location); or (nothing). Under the heading "IOR Context," Figure 2 also shows the conditions that were subtracted in the identity discrimination task to reveal the effect of repeating location

\section{S1 Presentation}

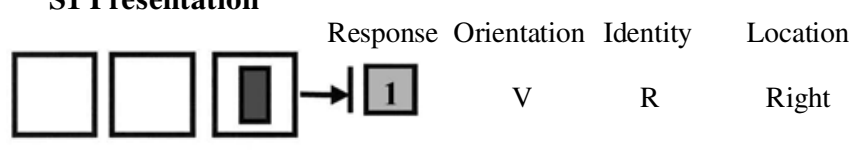

S2 Presentation
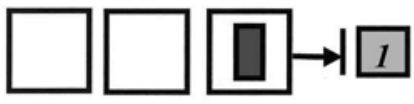

$\square \square$
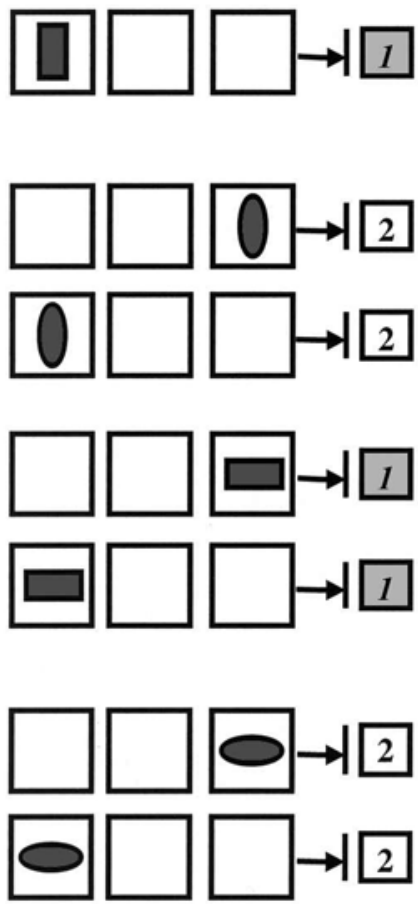

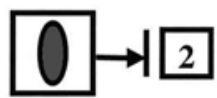

S2 Relative to S1

Location

V

$R$

$R$

V

V

$\mathrm{H}$

H

$R$

H

E

H
E

E

$R$

$R$
Right

Left

Right

Left

Right

Left

\section{Repetition IOR \\ Condition Context}
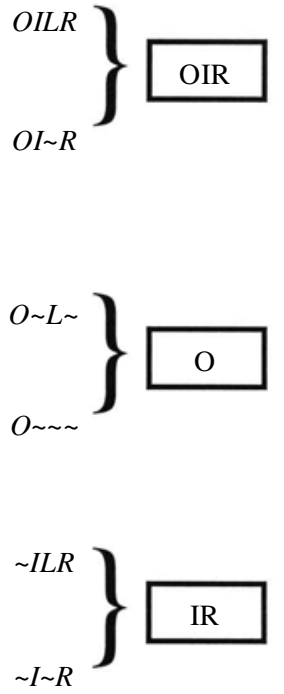

Figure 2. Experimental conditions in Experiment 1. 
while (1) stimulus identity, orientation, and response repeated (OIR-IOR), (2) stimulus orientation repeated ( $O$-IOR), (3) stimulus identity and response repeated (IR-IOR), or (4) no other stimulus or response dimensions ( -IOR) also repeated.

The repetition conditions for the orientation discrimination task were determined in an analogous way to those of the identity discrimination task. However, rather than the repetition of a response being redundant with the repetition of stimulus identity, the repetition of a response was redundant with the repetition of stimulus orientation. Thus, the repetition conditions were slightly different from those of the identity discrimination task. Whereas the repetition conditions for the identity discrimination task were $O I L R$, $O I \sim R, O \sim L \sim, O \sim \sim \sim, \sim I L R, \sim I \sim R, \sim \sim L \sim$, and $\sim \sim \sim$, the conditions for the orientation discrimination task were $O I L R, O I \sim R, O \sim L R$, $O \sim \sim R, \sim I L \sim, \sim I \sim \sim, \sim \sim L \sim$, and $\sim \sim \sim$.

For the Vanderbilt students, each block was 384 trials (6 repetitions of 4 S 1 stimuli $\times 2$ S1 locations $\times 4$ S2 stimuli $\times 2$ S2 locations). Owing to time restrictions for the Dalhousie students, each block was half this length ( 3 repetitions of 4 S1 stimuli $\times 2$ S1 locations $\times 4$ S2 stimuli $\times 2$ S2 locations). For the analyses, S1 identity and location were collapsed, thereby providing a maximum of 48 trials in each repetition condition for the Vanderbilt students and 24 trials for the Dalhousie students. ${ }^{3}$ The median RT for correct trials in each repetition condition was determined for each participant. ${ }^{4}$

Prior to performing in the experimental block proper, the participants performed a practice block consisting of the presentation of 20 trials drawn randomly from the experimental block. If the participants indicated feeling comfortable with the task demands following this practice block, they were allowed to proceed to the experimental block. Otherwise, they were allowed another practice block of 20 trials.

\section{Results}

The identity discrimination and orientation discrimination conditions will be considered separately. For both tasks, Table 3 shows the mean of the median correct RTs to $\mathrm{S} 1$, the mean of the median correct RTs to S2, the mean percent correct responses to S2 (\%Corr), and the standard error $(S E)$ associated with each of these measures.

In Table 3, an example of a trial type that would contribute to each repetition condition is shown (as in Figure 1). Under the Example heading, a vertically oriented rectangle represents a sample $S 1$ condition against which the S2 stimuli are compared (note that all the stimuli were yellow in the actual experiment). The black vertically and horizontally oriented rectangles and ellipses in the subsequent rows of the same column represent sample S2 conditions that exemplify the repetition condition. The "+" signs are used to indicate fixation but were not actually presented in the visual display (see Figure 1). The repetition conditions are outlined according to whether $\mathrm{S} 1$ and $\mathrm{S} 2$ repeated the same orientation $(O)$, identity $(I)$, and location $(L)$, and whether they required the same overt response $(R)$, in that order. As was described previously, the tilde $(\sim)$ is used as a placeholder to mark stimulus dimensions that did not repeat from $\mathrm{S} 1$ to $\mathrm{S} 2$.

The contextual IOR effects, shown in the last column, represent the effect of repeating versus alternating stimulus location when other stimulus-response dimensions also repeated. For the identity discrimination task, the difference between RTs to S2 in the OILR and the $O I \sim R$ conditions provides a measure of IOR when orientation, identity, and response repeated in the same versus a different location $(O I R-I O R)$; the difference between the $O \sim L \sim$ and the $O \sim \sim \sim$ conditions provides a measure of IOR when orientation repeated in the same versus a different location $(O$-IOR); the difference between the $\sim I L R$ and the $\sim I \sim R$ conditions provides a measure of IOR when identity and response repeated $(I R-\mathrm{IOR})$ in the

Table 3

Experiment 1: Results for the Identity and Orientation Discrimination Tasks

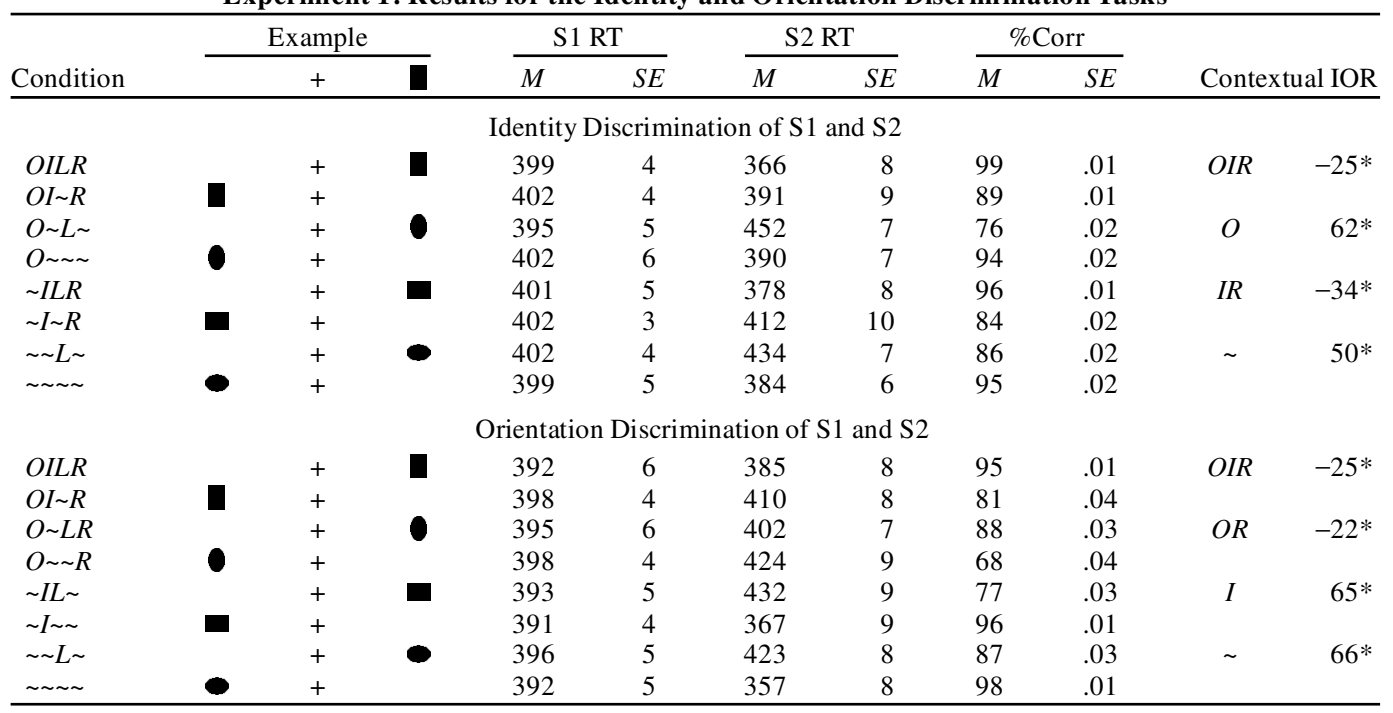

Note-\%Corr, mean percentage of correct responses to S2; O, orientation; I, identity; L, location; R, response; , placeholder. $* p<.05$. 
same versus a different location; and the difference between the $\sim L \sim$ and the $\sim \sim \sim$ conditions provides a measure of IOR when no stimulus dimension other than location repeated ( -IOR).

Analogously, for the orientation discrimination task, the difference between the OILR and the $O I \sim R$ conditions provides a measure of IOR when orientation, identity, and response repeated (OIR-IOR); the difference between the $O \sim L R$ and the $O \sim \sim R$ conditions provides a measure of IOR when orientation and response repeated (OR-IOR); the difference between the $\sim I L \sim$ and the $\sim I \sim \sim$ conditions provides a measure of IOR when identity repeated (I-IOR); and the difference between the $\sim \sim L \sim$ and the $\sim \sim \sim$ conditions provides a measure of IOR when no stimulus dimension other than location repeated ( - -IOR).

Identity discrimination. A repeated measures analysis of variance (ANOVA) was used to examine the $\mathrm{S} 2$ RTs as a function of repetition condition (OILR, OI R, $O \sim L \sim, O \sim \sim \sim, \sim I L R, \sim I \sim R, \sim \sim L \sim, \sim \sim \sim \sim)$. This analysis revealed a significant effect $[F(7,91)=26.24, M S=$ $12,245.82, p<.0001]$ that can be seen in Table 3 as overall longer RTs to S2 in the $O \sim L \sim$ and $\sim \sim L \sim$ conditions than in any other condition.

Contextual IOR effects were calculated from the S2 RT data, as was described above. Planned comparisons were used to examine the effect of repeating stimulus location in the context of repeating other stimulus-response dimensions (OIR-IOR, $O$-IOR, $I R$-IOR, -IOR). These analyses revealed significant facilitatory effects for repeating stimulus location when the discriminated stimulus dimension and required response also repeated (i.e., in the $O I R$-IOR and $I R$-IOR conditions) and significant inhibition when the discriminated stimulus dimension and required response did not repeat (i.e., in the $O$-IOR and $\sim$-IOR conditions). More particularly, for the OIRIOR condition, a significant 25 -msec facilitatory effect was revealed [OIR-IOR, $M=-25 \mathrm{msec} ; F(1,39)=9.57$, $M S=4,465.46, p<.003]$. This indicates that when $\mathrm{S} 2$ shared the same orientation and identity and required the same response as S1, RTs were faster if S2 occurred in the same rather than in a different location. Similarly, when S1 and S2 shared the same identity and required the same response, RTs were $34 \mathrm{msec}$ faster when S2 appeared in the same rather than in a different location as S1 [ $I R$-IOR, $M=-34 \mathrm{msec} ; F(1,39)=17.21, M S=$ $8,030.37, p<.0001]$. These effects are in sharp contrast to the significant $62-\mathrm{msec}$ IOR effect when orientation repeated in the same rather than in a different location $[O$-IOR, $F(1,39)=59.12, M S=27,585.70, p<.0001]$ and the 50-msec IOR effect when location alone repeated $[\sim-\mathrm{IOR}, F(1,39)=37.25, M S=17,381.53, p<.0001]$.

A one-way ANOVA of the mean percent correct S2 trials revealed a significant effect of repetition condition $[F(7,91)=25.99, M S=0.080, p<.0001]$. As can be seen in Table 3, it appears that this is closely tied to overall lower accuracy in the $O \sim L \sim$ condition, for which there were longer S2 RTs, relative to other conditions. This is the opposite pattern than would be expected if there were a speed-accuracy tradeoff. Planned comparisons confirmed that conditions showing RT facilitation for the repetition (rather than alternation) of stimulus location had greater overall accuracy, whereas conditions showing RT inhibition had lower overall accuracy. Accuracy was $10 \%$ higher when orientation, identity, and response repeated in the same rather than in a different location and was $12 \%$ higher when identity and response repeated in the same rather than in a different location. In contrast, there was an $18 \%$ IOR effect on accuracy when orientation repeated in the same versus in a different location and a 9\% IOR effect when location repeated alone.

In the target-target paradigm, responses to S1 are intended to generate IOR, and the speed of responses to S2 is used to reveal the effects of that inhibition (see Taylor \& Klein, 2000). This separation of cause and effect is successful only to the extent that the minimum interval between the $\mathrm{S} 2$ on trial $N-1$ and the $\mathrm{S} 1$ on trial $N$ are separated by an interval that extends beyond the typical window for observing the effects of IOR (up to $1,500 \mathrm{msec}$ ). In our experiment, the minimum interval between $\mathrm{S} 2$ on trial $N-1$ and the $\mathrm{S} 1$ on trial $N$ was the sum of the minimum RT to $\mathrm{S} 2$ on trial $N-1$, the $1,500-\mathrm{msec}$ intertrial interval, the 500-msec fixation interval, and the 200 -msec interval between the presentations of the fixation stimulus and S1. The fastest average S2 RT across all four experiments reported in this study was $302 \mathrm{msec}$ (in the Same condition of Experiment 3). Using this as a guideline and assuming that the participants did not pause between trials, it suggests that our shortest interval between the $\mathrm{S} 2$ of one trial and the $\mathrm{S} 1$ of the subsequent trial was $2,502 \mathrm{msec}$. This value is likely outside the temporal window for observing IOR. Furthermore, even if there were any general inhibitory effects that might potentially operate from $\mathrm{S} 2$ on trial $N-1$ to $\mathrm{S} 1$ on trial $N$, they would likely influence all repetition conditions equally and would, therefore, be of little threat to our conclusions. Even so, we determined whether effects on S1 RTs could be used to explain the IOR data that we obtained.

The critical question here is not so much whether RTs to $S 1 \mathrm{~s}$ were slowed as a result of a previous $S 2$ but, more critically, whether there were influences on the S1 RTs that impacted the IOR effects we observed for the S2 RTs within the same trial. If S1 RTs were systematically slowed as a result of sharing characteristics with the S2 of the preceding trial and if these slowed RTs influenced the RTs to the S2s on the same trial, we should see that in correlations between the S1 and S2 RTs that were produced within trials. In fact, the magnitude of the contextual IOR effects revealed by RTs to S2 were unrelated to the speed of responding to the S1 stimulus. The S1 RTs were dummy-coded according to the $\mathrm{S} 2$ condition that they preceded. Differences between conditions were cal- 
culated to determine a contextual effect in the same manner as had been done for S2 RTs. For example, the difference between the S1 RTs in the OILR and the OI R conditions (i.e., $O I L$-IOR) was regressed on the difference between the S2 RTs for the same conditions. The same was done for each contextual IOR condition. None of the correlations even approached significance: OIL-IOR $[r=.12 ; t(12)=0.42, p>.68], I R$-IOR $[r=$ $.30 ; t(12)=1.10, p>.29], O$-IOR $[r=-.20 ; t(12)=$ $-0.71, p>.49], \sim-$ IOR $[r=.12 ; t(12)=0.43, p>.67]$.

Orientation discrimination. The one-way withinsubjects ANOVA used to analyze RTs to $\mathrm{S} 2$ as a function of repetition condition $(O I L R, \sim I L \sim, O \sim L R, \sim \sim L \sim, O I \sim R$, $\sim I \sim \sim, O \sim \sim R, \sim \sim \sim \sim)$ revealed a significant effect $[F(7,91)=$ $30.31, M S=10,964.10, p<.0001]$. As can be seen in Table 3, this stems from relatively faster RTs in the OILR, $I \sim \sim$, and conditions than in the other conditions.

Planned comparisons were used to examine contextual IOR effects. As with the identity discrimination condition, these comparisons revealed that when S2 repeated in the same rather than in a different location as $\mathrm{S} 1$, there was significant facilitation when the discriminated dimension (orientation) and required response also repeated and significant IOR when they did not. In particular, as compared with cases in which $\mathrm{S} 2$ was in a new location, when $\mathrm{S} 2$ repeated in the same location, there was a significant 25 -msec facilitatory effect when it also shared the same orientation, identity, and response as $\mathrm{S} 1$ [OILR-IOR, $F(1,91)=11.81, M S=4,270.93, p<.0009]$. Likewise, there was a significant 22 -msec facilitatory effect when S2 occurred in the same rather than in a different location as $\mathrm{S} 1$ and shared the same orientation and required response [OR-IOR, $F(1,91)=9.51, M S=$ $3,441.90, p<.003]$. These effects were in sharp contrast to the significant $66-\mathrm{msec}$ IOR effect when S1 and S2 shared the same identity $[I-\mathrm{IOR}, F(1,91)=83.25, M S=$ $30,116.50, p<.0001]$ and $65-\mathrm{msec}$ IOR effect when $\mathrm{S} 2$ repeated nothing other than $\mathrm{S} 1$ location [ -IOR, $M=$ $65 \mathrm{msec}, F(1,91)=84.96, M S=30,735.10, p<.0001]$.

None of the effects on S2 RT were in a direction consistent with speed-accuracy tradeoffs. An ANOVA of percent correct $\mathrm{S} 2$ responses revealed a significant effect of repetition condition (OILR, $\sim I L \sim, O \sim L R, \sim \sim L \sim$, $O I \sim R, \sim I \sim \sim, O \sim \sim R, \sim \sim \sim \sim)$ on accuracy $[F(7,91)=$ 21.77, $M S=0.153, p<.0001]$. Planned comparisons confirmed that the highest percent correct scores occurred in those contextual IOR conditions that had the longest RTs. When $\mathrm{S} 2$ shared the same identity, orientation, and required response as $\mathrm{S} 1$, there was an overall $14 \%$ increase in percent correct responses to S2 when it occurred in the same rather than in a different location $[F(1,91)=21.15, M S=0.149, p<.0001]$. Likewise, when S2 shared the same orientation and required the same response as S1, there was an overall $20 \%$ increase in correct responses to $\mathrm{S} 2$ when it occurred in the same rather than in a different location as $\mathrm{S} 1[F(1,91)=40.12$, $M S=0.282, p<.0001]$. When only identity repeated in the same location, there was an overall IOR effect on accuracy, with a decrease of $19 \%$ relative to when location alternated between $\mathrm{S} 1$ and $\mathrm{S} 2[F(1,91)=35.97, M S=$ $0.253, p<.0001]$. When no stimulus dimension other than location repeated, there was an $11 \%$ IOR effect on accuracy $[F(1,91)=11.85, M S=0.083, p<.0009]$.

As had been done for the identity discrimination task, we determined that the magnitude of the contextual IOR effects cannot be accounted for by the speed of responding to S1. The S1 RTs were dummy-coded according to the $\mathrm{S} 2$ condition that they preceded. Differences between conditions were calculated to determine a contextual effect in the same manner as that for S2 RTs. The difference scores for RTs to S1 were then regressed on the corresponding difference scores for RTs to S2. None of the correlations approached significance: $O I L-\mathrm{IOR}[r=$ $.004 ; t(12)=0.221, p>.82], O R$-IOR $[r=-.292$; $t(12)=-1.06, p>.30], I-$ IOR $[r=-.023 ; t(12)=$ $-0.079, p>.93], \sim-\mathrm{IOR}[r=-.087 ; t(12)=-0.304, p$ $>$.76].

\section{Discussion}

Experiment 1 is one of the first explorations of IOR in a target-target task that requires an object discrimination response. Confirming the existence of IOR, there was a large, inhibitory effect associated with repeating location alone. This demonstrates that location-based inhibitory effects do occur when overt responses are required to sequential targets. The magnitude of this effect on RTs was $50 \mathrm{msec}$ for the identity discrimination condition and $66 \mathrm{msec}$ for the orientation discrimination condition. When the nondiscriminated stimulus dimension also repeated, there continued to be a large locationbased inhibition. In the case of the identity discrimination condition, the magnitude of IOR increased from 50 to $62 \mathrm{msec}$ when stimulus orientation also repeated; in the case of the orientation discrimination condition, the magnitude of IOR remained unchanged $(65 \mathrm{msec})$. Regardless of the reason for this difference between the two discrimination conditions, the fact remains that there was a large magnitude IOR effect associated with repeating location alone or in combination with a repeated nondiscriminated stimulus dimension.

Although these findings demonstrate that IOR can, in fact, be observed for an object discrimination performed within a target-target paradigm, it is clear that the nature of the discrimination determines which conditions will suffer or benefit from repetition. In the identity discrimination condition, IOR occurred unless identity/response repeated; in the orientation discrimination condition, IOR occurred unless orientation/response repeated. In other words, a location-based inhibition occurred only so long as the discriminated dimension (identity on the one hand, orientation on the other) and associated response did not repeat. In fact, when the discriminated dimension and response did repeat, significant facilitation was observed. 
Our finding of repetition effects for object discriminations is reminiscent of similar effects reported for occurrence discriminations. Terry et al. (1994) presented participants with a continuous-responding task that required the participants to make manual localization responses to discriminated targets. Although they found no overall IOR effects (as can be seen in Table 1), they did observe a trend for IOR when the target stimulus changed and facilitation when the stimulus remained unchanged. Likewise, in a target-target task that required participants to manually localize a discriminated target identity, Pratt and Abrams (1999) observed a modulation of IOR by target repetition. Unlike in Terry et al.'s continuousresponding paradigm that suggested facilitation when a target identity repeated in the same location, Pratt and Abrams observed IOR when the same target and the distractor repeated. However, the magnitude of this effect was reduced (albeit slightly) when the target and distractor changed. Similar results have been reported by Milliken, Tipper, Houghton, and Lupiáñez (2000).

Our results extend the observation of identity repetition effects to an object discrimination in a target-target paradigm. Moreover, by separating the repetition of discriminated and nondiscriminated dimensions, our results go one step further in demonstrating that the critical context for observing location-based inhibitory tagging is the repetition of stimulus location in the absence of the repetition of the discriminated stimulus dimension or overt response. In other words, we have demonstrated that facilitation does not occur as a simple consequence of repeating any aspect of the target stimulus; it is the repetition of the discriminated dimension and/or response that is critically important.

\section{EXPERIMENT 2}

The results of Experiment 1 demonstrated that IOR occurs for object discriminations in a target-target paradigm, but only when there is no concomitant repetition of the discriminated stimulus dimension/response. Because the participants in Experiment 1 made overt buttonpress responses to both $\mathrm{S} 1$ and $\mathrm{S} 2$, the repetition of the discriminated stimulus dimension was necessarily confounded with the repetition of the overt response. This makes it unclear whether the facilitatory effects were due to the repetition of the discriminated stimulus dimension or to the repetition of the overt response. Experiment 2 addressed this issue by replicating the method of Experiment 1 while eliminating the response to $\mathrm{S} 1$.

\section{Method}

Participants. Eighteen Vanderbilt University undergraduate students and 10 Dalhousie undergraduate students, who had not participated in Experiment 1, volunteered in exchange for psychology class credit. As with Experiment 1, the participants were tested either individually or in pairs in a dimly illuminated room in a single experimental session that required approximately $1 \mathrm{~h}$ for the Vanderbilt students and $0.5 \mathrm{~h}$ for the Dalhousie students. All the participants reported normal or corrected-to-normal vision and were naive as to the experimental purpose.

Stimuli and Apparatus. The stimuli and apparatus were identical to those in Experiment 1.

Procedure. The only change from Experiment 1 was that the participants were required to respond only to S2. The participants were instructed that $\mathrm{S} 1$ was uninformative with respect to S2 and that they were to try to ignore it. If the participants struck a key during the presentation of this stimulus, they were given an $833-\mathrm{msec}$ error message that informed them of the mistake, and the trial was aborted and recycled later in the block.

Table 4

Experiment 2: Results for the Identity and Orientation Discrimination Tasks

\begin{tabular}{|c|c|c|c|c|c|c|c|c|}
\hline \multirow[b]{2}{*}{ Condition } & \multicolumn{2}{|c|}{ Example } & \multicolumn{2}{|c|}{ S2 RT } & \multicolumn{2}{|c|}{$\%$ Corr } & \multirow{2}{*}{\multicolumn{2}{|c|}{ Contextual IOR }} \\
\hline & + & $\mathbf{0}$ & $M$ & $S E$ & $M$ & $\overline{S E}$ & & \\
\hline \multicolumn{9}{|c|}{ Identity Discrimination of S2 } \\
\hline OIL & + & 口 & 499 & 24 & 95 & .01 & $O I$ & 4 \\
\hline$O I \sim$ & + & & 495 & 20 & 96 & .01 & & \\
\hline$O \sim L$ & + & 0 & 539 & 22 & 96 & .01 & $O$ & $34 *$ \\
\hline$O \sim \sim$ & + & & 505 & 22 & 96 & .01 & & \\
\hline$\sim I L$ & + & $\square$ & 513 & 23 & 95 & .01 & $I$ & $15 \dagger$ \\
\hline$\sim I \sim$ & $\square$ & & 498 & 21 & 96 & .01 & & \\
\hline$\sim \sim L$ & + & - & 533 & 19 & 96 & .02 & $\sim$ & $25 *$ \\
\hline$\sim \sim$ & - & & 508 & 24 & 97 & .01 & & \\
\hline \multicolumn{9}{|c|}{ Orientation Discrimination of S2 } \\
\hline OIL & + & 口 & 575 & 27 & 94 & .01 & OI & 1 \\
\hline$O I \sim$ & + & & 574 & 28 & 95 & .01 & & \\
\hline$O \sim L$ & + & 0 & 623 & 26 & 94 & .01 & $O$ & $29 *$ \\
\hline$O \sim \sim$ & + & & 594 & 27 & 92 & .02 & & \\
\hline$\sim I L$ & + & $\square$ & 603 & 21 & 94 & .01 & $I$ & $28 *$ \\
\hline$\sim I \sim$ & 口 & & 575 & 23 & 95 & .01 & & \\
\hline$\sim \sim L$ & + & 0 & 603 & 24 & 96 & .02 & $\sim$ & $42 *$ \\
\hline$\sim \sim$ & 0 & & 561 & 23 & 95 & .01 & & \\
\hline
\end{tabular}

Note-\%Corr, mean percentage of correct responses to S2; O, orientation; I, identity; L, location; $\sim$, placeholder. * $p<$ $.05 . \quad \dagger .05<p<.10$. 
Half of the participants from each university (Vanderbilt and Dalhousie) performed an identity discrimination of $\mathrm{S} 2$, and the other half performed an orientation discrimination. Using $O$ to refer to a repeated stimulus orientation, $I$ to refer to a repeated stimulus identity, and $L$ to refer to a repeated location, the eight repetition conditions for Experiment 2 were (1) $O I L$, (2) $O I \sim$, (3) $O \sim L$, (4) $O \sim \sim$, (5) $\sim I L,(6) \sim I \sim$, (7) $\sim L$, and (8) .

In all other respects, Experiment 2 was identical to Experiment 1.

\section{Results}

Table 4 shows the mean of the median RTs and associated $S E$ s for correct responses to $\mathrm{S} 2$ and the mean percent correct and associated $S E$ s for the identity discrimination task and for the orientation discrimination task. Labeling conventions are the same as described for Table 3 .

For the contextual IOR scores, the difference in RT between the $O I L$ and the $O I \sim$ conditions was taken as the IOR effect when orientation and identity both repeated (OI-IOR); the difference between the $O \sim L$ and the $O \sim \sim$ conditions was taken as the IOR effect when orientation repeated $(O$-IOR); the difference between the $\sim I L$ and the $\sim I \sim$ conditions was taken as the IOR effect when identity repeated (I-IOR); and the difference between the $\sim \sim L$ and the $\sim \sim$ conditions was taken as the IOR effect when nothing else repeated ( -IOR). Because responses never repeated, these contextual IOR effects are calculated in identical ways for the identity and the orientation discrimination tasks.

Identity discrimination task. A one-way ANOVA was performed on the S2 RTs, with repetition condition (OIL, OI , $O \sim L, O \sim \sim, \sim I L, \sim I \sim, \sim \sim L, \sim \sim \sim)$ as a withinsubjects variable. This analysis revealed a significant effect of repetition condition $[F(7,91)=6.83, M S=$ $3,796.48, p<.0001$ ], which can be seen in Table 4 as generally longer RTs for conditions in which location repeated than for conditionsin which location did not repeat.

Planned contrasts were used to examine contextual IOR effects $(O I$-IOR, $O$-IOR, $I$-IOR, $\sim-\mathrm{IOR})$ in the S2 RT data. These analyses revealed a significant $34-\mathrm{msec}$ IOR effect when orientation repeated [ $O$-IOR, $F(1,39)=$ $14.65, M S=132.56, p<.0002]$ and a significant 25 msec IOR effect when no stimulus dimension other than location repeated [ -IOR, $F(1,39)=8.01, M S=1,559.21$, $p<.006]$. The 15 -msec inhibitory effect of repeating stimulus location was only marginally significant when identity repeated alone [I-IOR, $F(1,39)=2.80, M S=$ $8,149.03, p<.098$ ] but did not differ from the (significant) 25 -msec $\sim$-IOR effect $(F<1)$. In stark contrast, the IOR effect associated with repeating both stimulus identity and orientation did not even approach significance (OI-IOR, $M=4 \mathrm{msec}, F<1)$.

None of the effects on the S2 RTs were associated with effects on the percent correct scores. There was no significant omnibus effect $(F<1)$ of repetition condition (OIL, OI , O L, O , IL, I , L, ) on overall accuracy. Planned comparisons confirmed no significant contextual IOR effects on accuracy (all $p \mathrm{~s}>.23$ ).

Orientation discrimination task. As with the identity discrimination task, a one-way ANOVA was per- formed on the RT data from the orientation discrimination task, with repetition condition $(O I L, O I \sim, O \sim L$, $O \sim \sim, \sim I L, \sim I \sim, \sim \sim L, \sim \sim \sim)$ as a within-subjects variable. This analysis revealed a significant effect of repetition condition $[F(7,91)=7.73, M S=5,902.33, p<.0001]$. This is seen in Table 4 as generally longer RTs for conditions in which location repeated than for conditions in which location did not repeat.

Planned comparisons revealed a significant 28-msec IOR effect when identity repeated [I-IOR, $F(1,39)=$ $7.20, M S=550.34, p<.009]$, a significant $29-\mathrm{msec}$ IOR effect when orientation repeated [O-IOR, $F(1,39)=$ $8.01, M S=6,117.81, p<.006]$, and a significant 42msec IOR effect when no stimulus dimensions other than location repeated [ -IOR, $F(1,39)=15.73, M S=$ $12,011.37, p<.0001]$. There was no significant effect $(M=1 \mathrm{msec})$ of repeating stimulus location in conjunction with the repetition of both stimulus identity and orientation $(O I$-IOR, $F<1)$.

None of the effects in RTs to S2 were associated with effects on the percent correct scores. An omnibus analysis revealed no significant effects of repetition condition $(O I L, O I \sim, O \sim L, O \sim \sim, \sim I L, \sim I \sim, \sim \sim L, \sim \sim \sim)$ on accuracy $(F<1)$. Likewise, none of the planned comparisons examining percent correct for each contextual IOR condition revealed any significant effects (all $F_{\mathrm{S}}<1$ ).

\section{Discussion}

The purpose of Experiment 2 was to determine whether the facilitatory effects observed in Experiment 1 were due to the repetition of the discriminated stimulus dimension and/or to the repetition of an overt response. In this regard, the results of Experiment 2 are unambiguous: The significant facilitatory effects observed in Experiment 1 depended on response repetition (see also Hommel, 1998). When motor output was required only in response to $\mathrm{S} 2$, the facilitatory effect that had been observed for the $O I R$-IOR condition of Experiment 1 disappeared (in the $O I$-IOR condition of Experiment 2) for both the identity discrimination $(4 \mathrm{msec})$ and the orientation discrimination $(1 \mathrm{msec})$ tasks. Likewise, the facilitatory effect that had been observed in Experiment 1 when the discriminated stimulus dimension repeated in the same location (IR-IOR for the identity discrimination task and $O R$-IOR for the orientation discrimination task) reversed to become inhibitory in the comparable conditions of Experiment 2 (I-IOR for the identity discrimination and $O$-IOR for the orientation discrimination). Although the magnitude of the $I$-IOR effect in the identity discrimination condition was only marginally significant, it did not differ from the significant $25-\mathrm{msec}$ "pure" IOR effect in the -IOR condition. As such, it seems reasonable to consider that the failure to reach significance likely reflects a Type II error. The magnitude of the effect in the $O$-IOR orientation discrimination condition was $29 \mathrm{msec}$ and significant.

Insight into the conditions that govern the occurrence of IOR can be gained by comparing the pattern of results with 
that of Experiment 1. An important issue is whether the facilitatory effects in Experiment 1 reflected no locationbased inhibitory tagging when a discriminated target dimension repeated or whether IOR might have occurred but been masked by a facilitatory effect owing to the repetition of an overt response. On this issue, our results are also clear. In the identity discrimination condition, facilitation was observed in Experiment 1 in the $O I R$-IOR and $I R$-IOR conditions. When the repetition of an overt response was eliminated in Experiment 2, there was no effect (facilitatory or inhibitory) in the $O I$-IOR condition, but the results tended towards inhibition in the I-IOR condition. Similarly, in the orientation discrimination condition, facilitation was observed in Experiment 1 in the $O I R$-IOR and $O R$-IOR conditions. And when the repetition of an overt response was eliminated in Experiment 2, there was no effect (facilitatory or inhibitory) in the $O I$-IOR condition, but there was significant inhibition in the $O$-IOR condition. Taken together, these results suggest that IOR does, in fact, occur for the repetition of a discriminated dimension but was masked in Experiment 1 by a facilitatory effect associated with repetition of an overt response. In contrast, IOR does not occur when the discriminated and the nondiscriminated dimensions both repeat: The facilitatory effect observed in the $O I R$-IOR condition of Experiment 1 did not represent the masking of an otherwise inhibitory effect by a facilitatory response-related effect; instead, there was only a facilitatory effect owing to the repetition of an overt response.

The lack of IOR in Experiment 2 when identity and orientation both repeated suggests a role of object representations in modulating IOR (see also Lupiáñez \& Milliken, 1999). To recast the results of Experiment 2 in terms of stimulus changes rather than repetitions, it becomes clear that IOR occurred whenever there was a change in the target representation from S1 to S2. When orientation changed, IOR was observed (I-IOR, -IOR); when identity changed, IOR was observed $(O$-IOR, $\sim-$ IOR); when neither changed, IOR was not observed (OI-IOR). It seems reasonable to assume that the perception of an "object" depends on the conjunction of its defining features - in this case, orientation and identity (shape). To the extent that this is true, the results of Experiments 1 and 2 lead to the supposition that IOR is sensitive to changes in the "objectness" of peripheral stimuli. IOR appears to affect responses to S2 only when the $\mathrm{S} 2$ stimulus represents a change in the object characteristics presented as $\mathrm{S} 1$. This is intriguing because it suggests that IOR may slow responses only to a new perceptual object. And even more interesting is the implication that IOR may not be tagged until an evaluative comparison of $\mathrm{S} 1$ and $\mathrm{S} 2$ has been completed. This would argue that IOR operates at a relatively late representational stage within the information-processing stream and that it may be tagged to memorial representations of objects in space, rather than directly to the rep- resentation of a spatial location (see Milliken et al., 2000). Indeed, this would converge with recent findings that reveal a role for IOR in dissociating stimuli from their associated responses and from their lexical representations (Chasteen \& Pratt, 1999; Fuentes et al., 1999b).

\section{EXPERIMENT 3}

Taken together, the results of Experiments 1 and 2 demonstrate that IOR does not occur when the target stimulus remains unchanged from $\mathrm{S} 1$ to $\mathrm{S} 2$ in an object discrimination task. It is not clear, however, whether these apparent object-based effects depend on making an object discrimination. Does the finding of no IOR for a repeated target object depend on an explicit discrimination of the object that is being repeated, or would it also be observed when the physical characteristics of the target are task irrelevant? This is an important issue, because it has implications for our understanding of the conditions under which the expression of IOR may be modulated by object representations.

Experiment 3 conceptually replicated the design of Experiment 1, using a paradigm that made the visual characteristics of the target object irrelevant to the task. As in Experiment 1, we presented participants with two targets on every trial. These stimuli were one of two possible stimulus identities that could appear in one of four possible locations, two to the left and two to the right of a central fixation. This allowed us to examine the effects of repeating stimulus identity, location, and direction/ overt response in the context of a task that did not require the processing of any stimulus property other than its direction relative to fixation.

The fact that our methods required a spatial localization without a discrimination of target identity distinguishes Experiment 3 from previous investigations summarized in Tables 1 and 2. As an example, consider a study performed by Pratt and Abrams (1999). In a series of intricately designed experiments requiring stimulus localization, these authors systematically examined the effects of repeating discriminated stimuli in the same rather than in a different location. However, in all five of their experiments, $\mathrm{S} 2$ was presented simultaneously with a distractor. It was Pratt and Abrams's intention that participants process the identity of the S2 stimulus before reporting its location via an eye movement or buttonpress. This stands contrary to our purpose.

In that respect, our Experiment 3 is more similar to studies that have examined coincidental effects of repeating the same target identities in tasks that did not require the explicit processing of these identities. For example, Kwak and Egeth (1992) presented participants with a continuous-responding paradigm in which the participants were required to make a simple detection response to the onset of a target, regardless of its identity or location (see also the no-discrimination condition of 
S1 Presentation

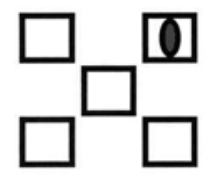

S2 Presentation
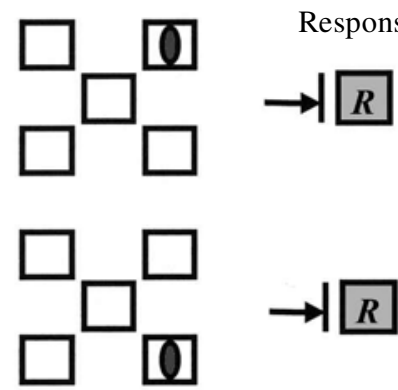

Same

S2
Response Stimulus Location Direction

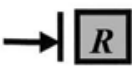
Same
$T R$
"Right”

BR

"Right”

BL

"Left"

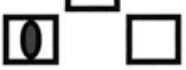

$\rightarrow \mathbf{L} \quad$ Same
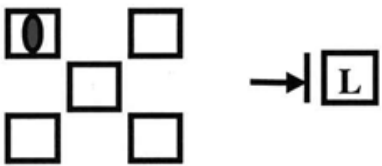

Same

TL

"Left"

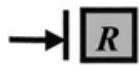

Different

TR

"Right"
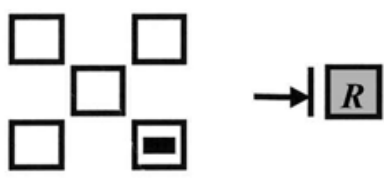

Different

BR

"Right"
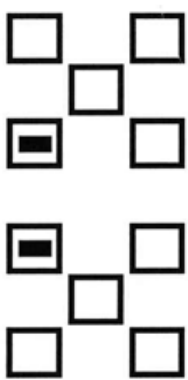

$\rightarrow \mathrm{L}$

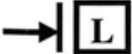

Different

Different
BL

"Left"

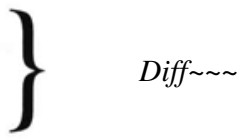

TL "Left"

Diff
Same DR

Diff LDR

Diff $\sim D R$

\section{Repetition Condition}

SameLDR

Same

Figure 3. Method used in Experiment 3.

Terry et al., 1994, Experiment 2). In different experiments, the stimuli on consecutive trials could repeat the same location, as well as a nonspatial feature (e.g., color). Participants were not asked to process the non- spatial feature and did not need to process this feature in order to perform the detection response. The authors examined whether there was an automatic inhibitory effect of repeating the nonspatial feature from trial $N-1$ to 
trial $N$ that was akin to the IOR effect observed when location repeated. There was not. IOR occurred for the repetition of target location, but there was no inhibitory effect that occurred with the repetition of target color, form, or orientation. Moreover, contrary to our findings with a discrimination task, in both a continuous-responding and a no-response-target paradigm, Kwak and Egeth revealed IOR whether or not target features repeated or changed across consecutive trials. These findings strongly suggest that the modulation of IOR by repeated object representations may be specific to tasks that require the discrimination of target features.

Experiment 3 determined whether this conclusion would hold for spatial localization responses made within the context of a novel target-target paradigm that allowed the effect of repeated location to be disentangled from the effect of a repeated direction. Our main interest was in determining whether a repeated target stimulus would continue to result in a lack of IOR (as in Experiments 1 and 2) or whether IOR in a nondiscrimination task would be immune to the effects of target repetition. The second goal of Experiment 3 was to provide a measure of overall IOR effects as a function of whether the S2 stimuli (regardless of identity) repeated the same location and/or the same direction/response as S1. This was intended to provide us with a sense of how IOR is tagged, using our novel methods.

\section{Method}

Participants. Eighteen Vanderbilt University undergraduate students who had not participated in Experiments 1 or 2 volunteered in exchange for credit toward their introductory psychology grade. As with the previous experiments, each participant was tested individually or in pairs in a dimly illuminated room in a single experimental session that required approximately $1 \mathrm{~h}$. All the participants reported normal or corrected-to-normal vision and were naive as to the experimental purpose.

Stimuli and Apparatus. Stimuli, timing, and apparatus were identical to those in Experiment 1, with one exception. Rather than three outline boxes, there were five, arranged as depicted in Figure 3. The fixation box was centered on the computer screen. The four stimulus boxes were separated from the fixation box by $3.0^{\circ}$ of visual angle and from one another by $4.3^{\circ}$ of visual angle center to center. The corners of the outside boxes were aligned on imaginary diagonals drawn corner to corner through the center of the fixation box. Ellipses and rectangles were clearly distinguishable.

Procedure. Both S1 and S2 were equally likely to appear in any one of the four stimulus boxes surrounding the fixation box. To maintain a reasonable number of trials, the increased number of stimulus positions was countered by a reduced set; rather than presenting participants with a four-identity pool, we presented them with only two stimulus identities. Six groups of 3 participants each were presented with one of the six combinations that result from all possible pairings of the four identities in Experiments 1 and 2: VE/HE, VE/VR, VE/HR, HE/VR, HE/HR, or VR/HR. The multiple pairing of stimulus identities was intended to increase the generalizability of our results and to provide a measure of the effects of changing versus repeating the same stimulus; the participants were not required to attend to nonspatial stimulus dimensions in order to perform the required task.

Rather than respond on the basis of identity, all the participants were required to make a spatially compatible buttonpress response to localize S1 and then S2, regardless of the physical properties or exact locations of the stimuli. They did so by pressing the "1" to indicate that the stimulus appeared to the left of the fixation box and the " 2 " to indicate that it appeared to the right of the fixation box. Errors and associated error messages were the same as those described for Experiment 1.

Table 5

Results for Experiment 3: Direction Discrimination of S1 and S2

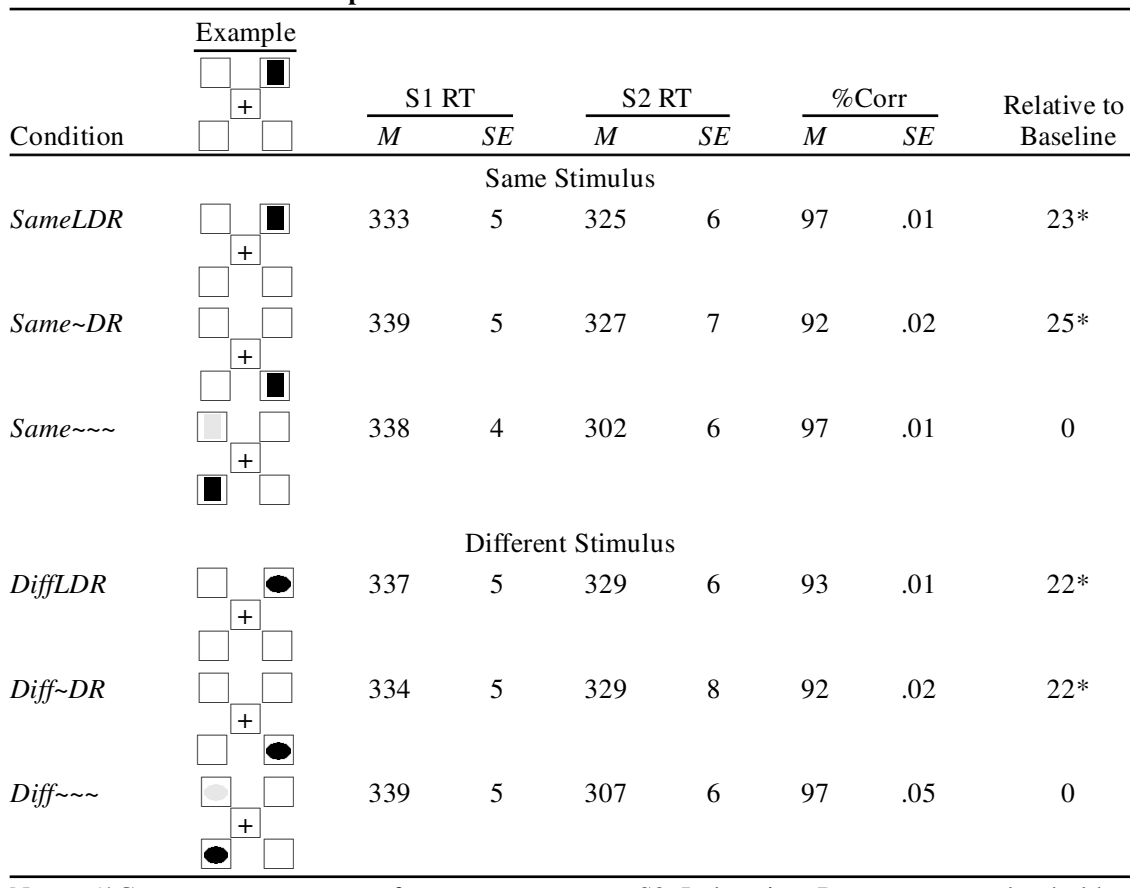

Note-\%Corr, mean percentage of correct responses to S2; L, location; R, response; , placeholder. $* p<.05$. 
The participants performed an experimental block of 384 trials (6 repetitions of $2 \mathrm{~S} 1$ stimuli $\times 4 \mathrm{~S} 1$ locations $\times 2 \mathrm{~S} 2$ stimuli $\times 4$ $\mathrm{S} 2$ locations). The data were collapsed across the six pairings of $\mathrm{S} 1 / \mathrm{S} 2$ stimulus identities, wherein the repetition of the same stimulus (same) was distinguished from a change in stimulus from $\mathrm{S} 1$ to $\mathrm{S} 2$ (different).

Using an example of a single S1, Figure 3 depicts the six repetition conditions that resulted from the presentation of S2. This figure depicts $\mathrm{S} 1$ as a vertically oriented rectangle presented in the top right stimulus box, relative to fixation. Using the key designations adopted in the present study, a press of the right key ("2" on the numeric keypad) is shown to report a stimulus to the right of fixation, and a press of the left key (" 1 " on the numeric keypad) is shown to report a stimulus to the left of fixation. Following the presentation of $\mathrm{S} 1$, one of two different $\mathrm{S} 2$ identities could be presented to one of the four stimulus boxes surrounding fixation. The examples shown in this figure are vertically oriented ellipses and horizontally oriented rectangles (under the label "S2 Presentation"). Relative to $\mathrm{S} 1, \mathrm{~S} 2$ could repeat the same stimulus (same, different) and could occur in the same location $(L)$ and in the same direction $(D)$ and could require the same localization response $(R)$. This resulted in six repetition conditions.

For the ease of presentation, repetition conditions will be identified according to whether the same or a different stimulus was presented as S1 and S2 (Same, Diff) and whether S2 repeated in the same location $(L)$ as $\mathrm{S} 1$ and/or in the same direction $(D$; i.e., to the left or right of fixation), in that order. To capture the fact that the participants could repeat the same overt response to both S1 and $\mathrm{S} 2$, this information is appended to the repetition condition labels. As in Experiments 1 and 2, a tilde ( ) will be used as a symbolic placeholder for conditions that did not repeat from S1 to S2. Using this terminology, the repetition conditions for Experiment 3 were: SameLDR, Same DR, Same , DiffLDR, Diff DR, Diff . Note that the repetition of location obligates the repetition of direction and response and that the repetition of direction obligates the repetition of response.

\section{Results}

Table 5 shows the mean of the median RTs to S2, the mean of the median RTs to S1, the percent correct S2 responses, and the $S E$ s of these measures, as a function of $\mathrm{S} 1 / \mathrm{S} 2$ stimulus relation (same, different), and repetition condition $(L D R, \sim D R, \sim \sim)$. An example of a trial type that would contribute to each repetition condition is shown. The empty boxes represent the spatial layout of the task; the center box with the cross represents the central fixation (note that a cross was not actually presented). The $\mathrm{S} 1$ in the example is a vertically oriented rectangle in the top right location, to which the participants would have been required to make a buttonpress with the right index finger. The stimuli in the following rows of the same column represent sample S2s that exemplify the repetition conditions. Note that the $\sim \sim$ conditions show both gray and black sample stimuli; this is intended to communicate the fact that a stimulus in either of the locations depicted would contribute to the repetition condition.

The RTs to S2 were analyzed in a repeated measures ANOVA, with stimulus relation and repetition condition as factors. This analysis revealed a significant main effect of stimulus relation $[F(1,17)=6.47, M S=367.62$, $p<.02]$, which did not interact with repetition condi- tion $(F<1)$, for which there was a significant main effect $[F(2,24)=10.82, M S=6,282.66, p<.0002]$.

Despite the nonsignificant omnibus ANOVA, planned comparisons were performed on differences between repetition conditions for the same and different stimulus relations (Keppel, 1982). The magnitude of these effects did not differ between same and different stimulus relations: $L D R-\sim \sim, L D R-\sim D R$, or $D R-\sim \sim ~($ all $F \mathrm{~s}<1$ ). In particular, comparisons of the $L D R$ and the $\sim \sim$ conditions revealed a significant 23 -msec effect for the same stimulus relation $[F(1,34)=58.06, M S=4,560.95, p<$ $.001]$ and a significant 22-msec effect for the different stimulus relation $[F(1,34)=53.70, M S=4,218.63, p<$ $.001]$. This indicates that whether or not the target object repeated, there was significant inhibition associated with repeating stimulus location/direction/response. Importantly, this effect could not be accounted for, in whole or in part, by the repetition of stimulus location; the difference between the $L D R$ and the $\sim D R$ conditions isolated only a 2-msec effect of repeating location when the repetition of stimulus direction and response were held constant in the same condition and no difference at all in the different condition (both $F_{\mathrm{S}}<1$ ). In contrast, there was a significant $25-\mathrm{msec}$ inhibitory effect associated with the combined repetition of stimulus direction and response [when stimulus location did not repeat, $\sim D R$ vs. $\sim \sim ; F(1,34)=72.88, M S=5,722.95, p<.001]$ in the same condition; the effect was $22 \mathrm{msec}$ for the comparable comparison in the different condition $[F(1,34)=$ $55.42, M S=4353.69, p<.001]$. This suggests that direction/response repetition was the critical factor for obtaining an inhibitory effect in this experiment.

An analysis of accuracy contradicted any suggestion of a speed-accuracy tradeoff. An ANOVA with stimulus relation (same, different) and repetition condition $(L D R$, $\sim D R \sim, \sim \sim \sim)$ as factors revealed only a significant effect of repetition condition $[F(2,34)=11.08, M S=0.026$, $p<.0002$; other $p$ s $>.16]$. This effect was due to overall greater accuracy in exactly those conditions that showed the fastest S2 RTs: The mean S2 RT of $305 \mathrm{msec}$ in the condition was associated with $97 \%$ accuracy; the mean S2 RT of $326 \mathrm{msec}$ in the $L D R$ condition was associated with $95 \%$ accuracy; and the mean S2 RT of $328 \mathrm{msec}$ in the $\sim D R$ condition was associated with $92 \%$ accuracy. For the same stimulus relation, relative to the $92 \%$ accuracy in the $\sim D R$ condition, the $L D R[F(1,34)=$ $10.18, M S=0.03, p<.003]$ and $\sim[F(1,34)=10.18$, $M S=0.03, p<.003]$ conditions were both significantly more accurate $(M=97 \%$ for both). For the different stimulus relation, relative to the $97 \%$ accuracy in the $\sim \sim$ condition, the $L D R[M=93 \% ; F(1,34)=4.99, M S=$ $0.014, p<.032]$ and $\sim D R[M=92 \% ; F(1,34)=8.25$, $M S=0.023, p<.007]$ conditions were both less accurate. There was no difference between the $L D R$ and the $\sim D R$ conditions $(F<1)$. None of the calculated differences between repetition conditions were significantly different when the same and the different stimulus rela- 
tions were compared: $L D R-\sim \sim \sim, \sim D R-\sim \sim \sim, L D R-\sim D R$ (all $p \mathrm{~s}>.08$ ).

As for Experiment 1, we determined whether the effects observed in S2 RTs were accountable by variations in S1 RTs. Differences in S1 RTs were taken between pairs of dummy-coded conditions and regressed on analogous differences in S2 RTs. The RT correlations between $\mathrm{S} 1$ and $\mathrm{S} 2$ were not significant for any of the difference scores: $L D R$ versus $\sim \sim[r=-.06 ; t(16)=$ $-0.22, p>.83], L D R$ versus $\sim D R[r=-.39 ; t(16)=$ $-1.71, p>.10]$, or $\sim D R$ versus $\sim \sim[r=.07 ; t(16)=$ $0.30, p>.80]$.

\section{Discussion}

In light of the results obtained for Experiment 1, Experiment 3 suggests that the modulation of IOR by a repeated target stimulus occurs only when nonspatial features serve as the basis for responding. When the participants were required to localize, without discriminating, $\mathrm{S} 1$ and $\mathrm{S} 2$, there was no effect of repeating versus changing the target stimulus. Our results replicate and extend those of Kwak and Egeth (1992).

On this issue of IOR more generally, the results of Experiment 3 also point to the role of repeated direction and/or response in establishing inhibition. Relative to when nothing repeated, responses to $\mathrm{S} 2$ were $25 \mathrm{msec}$ slower for the same stimulus relation and $22 \mathrm{msec}$ slower for the different stimulus relation when direction and response repeated; this effect was unchanged (23 and

Table 6

Results for Experiment 4: Direction Discrimination of S1

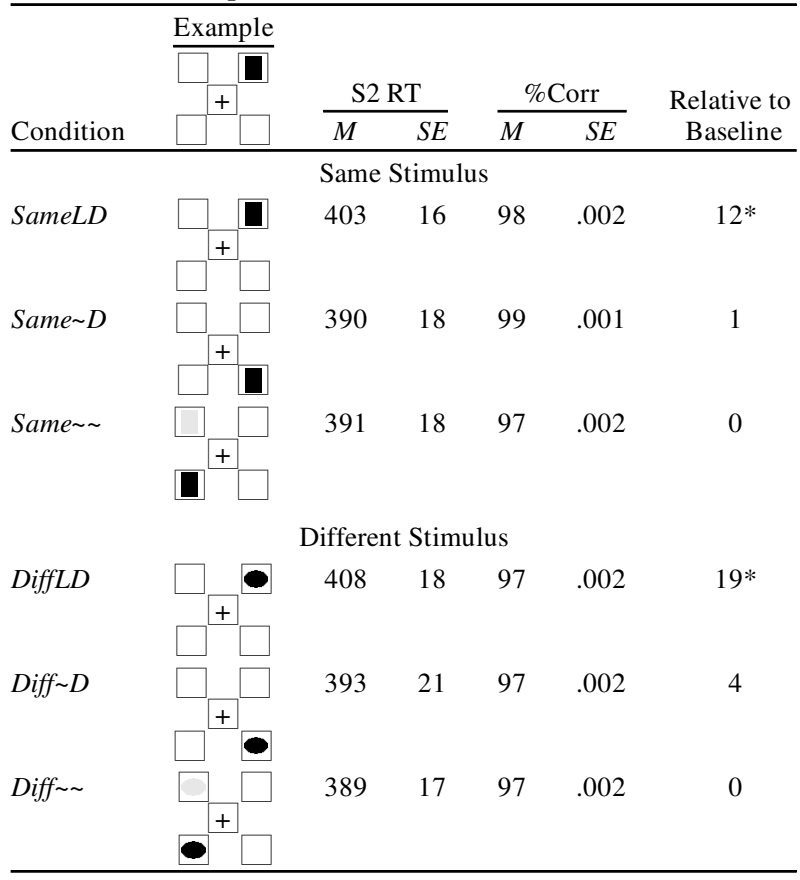

Note-\%Corr, mean percentage of correct responses to S2; L, location; $\mathrm{D}$, distance; $\sim$, placeholder. ${ }^{*} p<.05$.
$22 \mathrm{msec}$, respectively) when location also repeated. In a typical IOR task, target location and direction are entirely confounded. It has generally been assumed that IOR represents slowed responses to stimuli that repeat in the same location. However, our findings introduce the possibility that IOR may, instead, represent slowed responses to stimuli that repeat in the same direction - at least in the paradigm we have used. Of course, by virtue of the fact that our participants were required to indicate the direction of the S1 and S2 stimuli, it is possible that the inhibitory effects we observed were due to the repetition of an overt response, rather than to the repetition of stimulus direction per se. In Experiment 4, this possibility was examined.

\section{EXPERIMENT 4}

Experiment 4 repeated Experiment 3, except that the participants were required to respond only to $\mathrm{S} 2$. This allowed us to determine whether any effects of repeating stimulus identity would emerge when response repetition was eliminated and also to examine the effects of repeating stimulus location versus direction.

\section{Method}

Participants. Eighteen Vanderbilt University undergraduate students who had not participated in Experiments 1-3 volunteered in exchange for credit toward their introductory psychology grade. Each participant was tested individually in a dimly illuminated room in a single experimental session that required approximately $1 \mathrm{~h}$. All the participants reported normal or corrected-to-normal vision and were naive as to the experimental purpose.

Stimuli and Apparatus. Stimuli, timing, and apparatus were identical to those in Experiment 3.

Procedure. The procedure was identical to that in Experiment 3, except that the participants were instructed to respond only to S2. Errors and error messages were the same as those described for Experiment 2.

Using a terminology similar to that in Experiment 3, the repetition conditions were SameLD, Same D, Same , DiffLD, Diff D , and Diff . The differences between pairs of conditions were used to determine the effects of repeating stimulus, location, and/or direction. Note that the repetition of location obligates the repetition of direction, but not vice versa.

\section{Results}

Table 6 shows the mean of the median RTs to S2, the mean percent correct trials, and the standard error of both these measures, as a function of repetition condition. The labeling conventions are the same as those described for Table 5 .

The RTs to $\mathrm{S} 2$ were analyzed in a repeated measures ANOVA, with stimulus relation (same, different) and repetition condition $(L D, \sim D, \sim \sim)$ as factors. This analysis revealed only a significant main effect of repetition condition $[F(2,34)=6.02, M S=2,541.98, p<.006$; other $p \mathrm{~s}>$.26]. As had been done for Experiment 3, planned comparisons were used to examine differences between repetition conditions for the same and different stimulus relations. The difference between the $L D$ and 
the $\sim$ conditions revealed a significant 12 -msec difference for the same stimulus relation $[F(1,34)=11.61$, $M S=1,125.69, p<.002]$ and a significant 19 -msec difference for the different stimulus relation $[F(1,34)=$ $32.77, M S=3,176.94, p<.001]$. These two values did not differ significantly from one another $[F(1,17)=$ $2.565, M S=596.42, p>.12]$. This indicates that, for both stimulus relations, there was significant inhibition associated with repeating stimulus location/direction. Taking the difference between the $L D$ and the $\sim D$ conditions as the effect of repeating location when the repetition of direction was held constant, there was a significant 13 -msec effect for the same stimulus relation $[F(1,34)=14.96, M S=1,450.26, p<.001]$ and a significant 15 -msec effect for the different stimulus relation $[F(1,34)=22.21, M S=2,153.12, p<.001]$. Again, these values did not differ significantly $(F<1)$. This suggests that the effect of repeating location and direction (for both stimulus relations) may be accounted for entirely by the repetition of location. Indeed, this conclusion is supported by the fact that the repetition of direction alone resulted in no significant inhibition for either the same or the different stimulus relation, as revealed by the nonsignificant 1 - and 4-msec differences between the $\sim D$ and the $\sim \sim$ conditions (both $p \mathrm{~s}>.31$ ); the magnitude of these differences did not differ from each other $(F<1)$.

None of the effects on S2 RTs are consistent with speed-accuracy tradeoffs. An ANOVA, with stimulus relation (same, different) and repetition condition $(L D$, $\sim D, \sim \sim)$ as factors revealed no significant effects on overall percent correct (all $p \mathrm{~s}>.30$ ). Planned comparisons akin to those performed on the S2 RT data likewise revealed no significant effects (all $p \mathrm{~s}>.10$ ), except for a comparison of the $99 \%$ accuracy in the Same D condition to the $97 \%$ accuracy in the Same condition $[F(1,34)=4.86, M S=0.04, p<.034]$. None of the differences between stimulus relations were significantly different in the comparison of same and different stimulus relations (all $F \mathrm{~s}<1$ ).

\section{Discussion}

The results of Experiment 4 confirm those of Experiment 3 with respect to the role that repeated stimulus identities play in modulating spatial inhibition: There was no effect of repeating the same versus a different stimulus in our localization task. As such, these effects are consistent with almost countless observations of IOR for nondiscrimination tasks performed within the context of cue-target paradigms for which the cue and the target are physically different (e.g., beginning with Posner \& Cohen, 1984) and for which the cue and the target are physically identical (e.g., Tassinari \& Berlucchi, 1993; Taylor \& Klein, 2000). And, taken together, Experiments 3 and 4 strongly suggest that IOR was eliminated by repeated target stimuli in Experiments 1 and 2 precisely because discrimination of the target objects was used as the basis for responding.
The results of Experiment 4 also demonstrate that the apparent effect of repeating stimulus direction (as opposed to location) in Experiment 3 was likely due to the concomitant repetition of an overt response that reported direction. When a response was required only to S2, RTs were unaffected by the repetition of direction alone (Experiment 4). However, when direction repeated along with location, there was a 12-msec inhibitory effect for the same stimulus relation and a $19-\mathrm{msec}$ inhibitory effect for the different stimulus relation, relative to when nothing repeated.

\section{GENERAL DISCUSSION}

The issue of whether or not IOR occurs for target discriminations proves pivotal in theorizing about the mechanisms that may underlie this inhibitory effect. If IOR is postulated to reflect (either in whole or in part) slowed attentional reallocation to the inhibited location, it should impact the same target tasks that are sensitive to the influences of attentional allocation (see ReuterLorenz et al., 1996). As we described in our introduction, there is considerable evidence to support the influence of IOR on occurrence discriminations (e.g., Pratt, 1995; Pratt \& Abrams, 1999; Pratt et al., 1997). However, because these tasks are subject to motor bias effects, as well as to potential attentional influences associated with IOR, it is difficult to know whether this evidence is truly consistent with an attentional hypothesis. As such, it behooves researchers to use tasks that are relatively immune to motor effects of IOR that may otherwise be present. On the grounds that object discriminations serve this function, we noted that the evidence is mixed with regard to whether IOR occurs for these tasks. Certainly, there is strong evidence (see Table 2) that IOR can be obtained for object discriminations when a cuetarget paradigm is employed. However, when a continuousresponding paradigm is employed, negative results are obtained. The goal of our study was to clarify this discrepancy in the literature by using both target-target and no-response-target paradigms to parcel out interactions between spatial and object-based representations that might have interfered with obtaining IOR for object discriminations in continuous-responding paradigms, but not in cue-target paradigms. Before considering how our study addressed these issues, we will provide a brief summary of our main findings.

In Experiment 1, we observed that when the participants were required to process the identity or orientation of the S1-S2 stimuli, large facilitatory effects emerged in those conditions that repeated the overt response. When we focused on IOR independently of repeated motor output, in Experiment 2, we concluded that slowed responding to stimuli that repeated in the same location occurred only when some aspect of the stimulus object (orientation, identity) changed; when orientation and identity both repeated in the same location, there was no inhibition. In Experiments 3 and 4, we confirmed that 
this modulation of inhibitory spatial tagging by the repeated presentation of a target was task dependent. Using a localization task that could be performed without reference to any aspect of the target other than its direction relative to fixation, we observed that the repetition of the same target object played no role in modulating the occurrence of IOR. And yet, despite no role of repeated targets, there continued to be strong locationbased effects on S2 RTs (Experiment 4) and an effect of repeated stimulus direction when this repetition occurred in conjunction with a repeated overt response (Experiment 3). The fact that location, rather than direction, appeared to be the determinant of IOR when only one response was required is consistent with the notion that spatial location is the indispensable attribute in vision (e.g., Kubovy, 1988).

\section{IOR for Object Discriminations: Reconciling Discrepancies in the Literature}

Returning to the main purpose of our exploration, we sought to determine why IOR might occur for object discriminations made within cue-target paradigms, but not for those made within continuous-responding paradigms. Our goal was to clarify the issue of whether we can conclude that IOR is observed for object discriminations generally, or whether it is observed for object discriminations only in circumscribed situations (i.e., only within cue-target paradigms). As we noted in the introduction to our experiments, in a cue-target paradigm that requires participants to report a discriminated target feature, the only characteristic that can repeat from cue to target is location; the cue (by our classification) is visually distinct from the target and does not require an overt response. In this respect, the results from cue-target paradigms map most closely onto the $\sim$-IOR condition of Experiment 2, for which we observed a robust 25-msec IOR effect when identity was discriminated and a 42-msec IOR effect when orientation was discriminated. This is consistent with the observation of IOR for object discriminations following an onset cue in a cue-target paradigm (see Table 2).

With respect to IOR in continuous-responding paradigms, our results suggest that IOR in such a task may not be observed if the effects of repeating versus changing a spatial location are collapsed over changes or repetitions in the target object or overt response. Indeed, despite claims against IOR for (occurrence and object) discriminations, Terry et al.'s (1994) data spoke to the possible mitigating role of target repetitions on IOR. In an object discrimination, Terry et al. presented participants with a target/nontarget choice discrimination task that enabled the isolation of "pure" location-based inhibitory effects from the facilitatory effects associated with repeating a target identity or response. Consistent with our observation of facilitatory effects owing to the repetition of an overt response in a target-target task, Terry et al. reported that when target identity and response both repeated in their continuous-responding par- adigm, RTs were $47 \mathrm{msec}$ faster if they did so in the same rather than in a different location. Likewise, when response repeated alone, they found that RTs were $24 \mathrm{msec}$ faster if the stimulus was presented in the same rather than in a different location. In contrast, akin to our - -IOR conditions, which showed robust IOR effects, Terry et al. reported that when a target followed a nontarget, so that neither identity nor response repeated, RTs were $22 \mathrm{msec}$ slower if the stimulus followed in the same rather than in a different location. (The effects were in the same direction, but of a lesser magnitude for the nontargets.) Thus, in addition to converging with the positive results reported for object discriminations within cue-target paradigms, our results also converge with results that have been obtained for object discriminations within continuous-responding paradigms. Moreover, our results clearly demonstrate that IOR is obtained for object discriminations made within paradigms that require overt responses to sequential targets. The only stipulation is that the determination of IOR effects in such a situation must not collapse over repetitions of the target object or overt response: Facilitatory effects are produced by response repetitions, and target repetitions may be immune to the effects of IOR.

Our finding of interactions between location and object repetitions also helps qualify other thoughts on why IOR might occur for object discriminations made within cue-target paradigms, but not for those made within continuous-responding paradigms. It was postulated that IOR may not occur in the latter because object discriminations fail to generate and/or produce IOR. Our use of a target-target paradigm in Experiment 1 minimized the likelihood that targets were performing the dual role of both generating inhibition for the subsequent target and measuring inhibition from the preceding target. Despite this change in paradigm, as we noted above, when broken down according to repetition conditions, our results mapped nicely onto results reported by Terry et al. (1994) for object discriminations made within a continuousresponding paradigm. This suggests that there are no untoward effects associated with a repeated target presentation per se that interfere with IOR effects in continuousresponding paradigms. Object discriminations can generate IOR, and they are sensitive to the effects of this inhibition. However, the IOR does not occur as a simple consequence of repeating a stimulus in the same location; the target object and overt response must change.

Likewise, the results from Experiment 1 demonstrate that IOR is not prevented by the demand to execute two successive responses per se. We did observe IOR in a target-target paradigm in which the participants were required to execute responses to both $\mathrm{S} 1$ and S2. However, IOR did not occur if the same overt response repeated (Experiment 1) or if the target stimulus remained unchanged (Experiment 2). This latter result demonstrates that spatial inhibitory tagging is modulated by interactions with nonspatial features of the target (i.e., the discriminated and nondiscriminated target dimensions). 
And furthermore, this effect is specific to tasks that require an object discrimination; repeating a target stimulus had no influence in a task that did not require a visual discrimination (Experiments 3 and 4).

With regard to the main purpose of our study, the conclusion is that, like occurrence discriminations, locationbased inhibitory tagging can occur for object discriminations across a variety of paradigms. However, as is also the case with occurrence discriminations (see Pratt \& Abrams, 1999; Terry et al., 1994), the examination of IOR effects on object discriminations requires sensitivity to conditions under which the repetition of nonspatial target characteristics may mask or prevent the spatial inhibition.

\section{Mechanisms of IOR in Object Discrimination Tasks}

Because targets in Experiments 1 and 2 were as likely to require a left buttonpress as a right buttonpress, regardless of stimulus location, any motor influences on IOR that may have been present were undoubtedly small (see note 1). As such, the fact that location-based IOR occurred for object discriminations (at least in some conditions) would seem, at first blush, to be broadly consistent with attentional views of IOR, which postulate a role of slowed attentional reorienting in establishing inhibition for a previously stimulated location. However, it is difficult to conceive of an attentional account that would accommodate the finding of slowed RTs when a new target object repeats in the same rather than in a different location, but not when the same target object repeats. Determining whether a target object is the same or different requires some allocation of attentional resources to the target location. It is, therefore, illogical to suggest that attention is slowed to return to a previously stimulated location when it is occupied by a repeated target, but not when it is occupied by a changed target; whether repeated or changed, attention would have had to be deployed to the previously stimulated location in order to make this evaluation in the first place.

An alternative to an attention account suggests that participants engage a response heuristic for dealing with stimulus repetitions. If participants are sensitive to the fact that the stimulus object did not change from S1 to $\mathrm{S} 2$, it would be reasonable for them to assume that the associated response also did not change. The repetition of the stimulus object in Experiment 1 would therefore encourage the participants to make the same response to $\mathrm{S} 2$ as they had just made to $\mathrm{S} 1$, effectively bypassing the need to perform stimulus-response mapping and thereby speeding RTs. Because no overt response was made to S1 in Experiment 2, such an account would have to assume that an implicit response was generated to $\mathrm{S} 1$ and repeated overtly to $S 2$ when the stimulus object repeated.

The fact that the benefit of a hypothesized response heuristic was smaller in Experiment 2 than in Experiment 1 (as evinced by the change from significant facilitation in the $O I R$-IOR condition of Experiment 1 to no effect in the $O I$-IOR condition of Experiment 2) would require the further assumption either that the participants did not generate an implicit response to $\mathrm{S} 1$ on every trial of Experiment 2 or that the generation of an implicit response produced a less robust effect than did the execution of an overt response (as in Experiment 1). As well, this account has difficulty accommodating some of our findings. If the participants used a heuristic whose rule was "when everything remains the same, repeat the last implicit or overt response," we would have predicted an effect of repeating stimulus identity in Experiments 3 and 4 . Yet there was no such effect.

Our findings are more consistent with the view that a constructive retrieval process (see Milliken et al., 2000) is responsible for IOR in (occurrence and object) discrimination tasks. Extending Kahneman, Treisman, and Gibbs's (1992) notion of object files, Milliken et al. (2000) proposed that the emergence of facilitatory versus inhibitory effects depends on the efficiency with which episodic representations are newly made versus updated. When a target repeats in the same location, the existing object file is updated; when a target changes location, a new object file is created. Because attention is needed for the efficient integration of perceptual and memorial processes, the withdrawal of attention from the previously stimulated location means that updating the existing file for a target presented at this location is less efficient than making a new file for a target presented at a new location. However, visuospatial attentional allocation may not be the only factor that affects the efficiency of integration; "the inefficient spatial integration may be overridden by efficient integration based on stimulus dimensions other than spatial location" (Milliken et al., 2000, pp. 1293-1294). To the extent that this may be true, our findings from Experiments 3 and 4 clearly indicate that relative efficiencies associated with creating and maintaining object files do not influence tasks that require no discrimination.

Through the demonstration of a complex interplay between repeated target objects and repeated locations in object discrimination tasks, but not in nondiscrimination tasks, our results suggest that IOR in these two tasks may be mediated by different mechanisms. IOR in nondiscrimination tasks may be accounted for by some combination of attentional and motor effects. However, IOR in discrimination tasks may be more easily accounted for by constructive retrieval processes that interfere with IOR's being tagged indiscriminately to a repeated spatial location. Thus, our results open the door to further investigations into the nature and source of interactions related to the repetition of identity, location, and response and suggest that, through mechanisms associated with higher level representational systems (see Fuentes et al., 1999a), IOR may subserve efficient visual search in a flexible and adaptive way.

\section{REFERENCES}

Aвrams, R. A., \& Dob KIN, R. S. (1995). Inhibition of return: Effects of attentional cuing on eye movement latencies. Journal of Experimental Psychology: Human Perception \& Performance, 20, 467-477. 
Abrams, R. A., \& Pratt, J. (2000). Oculocentric coding of inhibited eye movements to recently attended locations. Journal of Experimental Psychology: Human Perception \& Performance, 26, 776-788.

Becker, L., \& Egeth, H. (2000). Mixed reference frames for dynamic inhibition of return. Journal of Experimental Psychology: Human Perception \& Performance, 26, 1167-1177.

Berger, A., Dori, H., \& Henik, A. (1999). Peripheral non-informative cues do induce early facilitation of target detection. European Journal of Cognitive Psychology, 11, 119-137.

Berger, A., \& HeniK, A. (2000). The endogenous modulation of IOR is nasal-temporal asymmetric. Journal of Cognitive Neuroscience, 12, 421-428.

Berlucchi, G., Chelazzi, L., \& Tassinari, G. (2000). Volitional covert orienting to a peripheral cue does not suppress cue-induced inhibition of return. Journal of Cognitive Neuroscience, 12, 648-663.

Berlucchi, G., Tassinari, G., Marzi, C. A., \& Di Stefano, M. (1989). Spatial distribution of the inhibitory effect of peripheral noninformative cues on simple reaction time to non-fixated visual targets. Neuropsychologia, 27, 201-221.

Buckolz, E., O’Donnell, C., \& McAuliffe, J. (1996). The Simon effect: Evidence of a response processing "functional locus." Human Movement Science, 15, 543-564.

Chasteen, A. L., \& Pratt, J. (1999). The effect of inhibition of return on lexical access. Psychological Science, 10, 41-46.

Cheal, M., \& Chastain, G. (1999). Inhibition of return: Support for generality of the phenomenon. Journal of General Psychology, 126, 375-390.

Danziger, S., \& Kingstone, A. (1999). Unmasking the inhibition of return phenomenon. Perception \& Psychophysics, 61, 1024-1037.

Danziger, S., Kingstone, A., \& SNyder, J. J. (1998). Inhibition of return to successively stimulated locations in a sequential visual search paradigm. Journal of Experimental Psychology: Human Perception \& Performance, 24, 1467-1475.

DownING, C. J. (1988). Expectancy and visual-spatial attention: Effects on perceptual quality. Journal of Experimental Psychology: Human Perception \& Performance, 14, 188-202.

Folk, C. L., Remington, R. W., \& Johnston, J. C. (1992). Involuntary covert orienting is contingent on attentional control settings. Journal of Experimental Psychology: Human Perception \& Performance, 18, 1030-1044.

Fuentes, L. J., Vivas, A. B., \& Humphreys, G. W. (1999a). Inhibitory mechanisms of attentional networks: Spatial and semantic inhibitory processing. Journal of Experimental Psychology: Human Perception \& Performance, 25, 1114-1126.

Fuentes, L. J., Vivas, A. B., \& Humphreys, G. W. (1999b). Inhibitory tagging of stimulus properties in inhibition of return: Effects on semantic priming and flanker interference. Quarterly Journal of Experimental Psychology, 52, 149-164.

Gibson, B. S., \& Amelio, J. (2000). Inhibition of return and attentional control settings. Perception \& Psychophysics, 62, 496-504.

Gibson, B. S., \& Egeth, H. (1994). Inhibition of return to object-based and environment-based locations. Perception \& Psychophysics, 55, 323-339.

Handy, T. C., Jha, A. P., \& Mangun, G. R. (1999). Evidence for an attention component in inhibition of return. Psychological Science, 10, 157-161.

Hawkins, H. L., Hillyard, S. A., Luck, S. J., Mouloua, M., DownING, C. J., \& WoodWARD, D. P. (1990). Visual attention modulates signal detectability. Journal of Experimental Psychology: Human Perception \& Performance, 16, 802-811.

Henderson, J. M., \& Macquistan, A. D. (1993). The spatial distribution of attention following an exogenous cue. Perception \& Psychophysics, 53, 221-230.

Hommel, B. (1998). Event files: Evidence for automatic integration of stimulus-response episodes. Visual Cognition, 5, 183-216.

Howard, L. A., LupiáñEz, J., \& Tipper, S. P. (1999). Inhibition of return in a selective reaching task: An investigation of reference frames. Journal of General Psychology, 126, 421-442.

IvANOFF, J., KLEIN, R. M., \& LuPIáÑEZ, J. (in press). Does inhibition of return interact with the Simon effect? Perception \& Psychophysics.

JoNIDES, J. (1981). Voluntary versus automatic control over the mind's eye's movement. In J. [B.] Long \& A. [D.] Baddeley (Eds.), Attention and performance IX (pp. 187-203). Hillsdale, NJ: Erlbaum.

JORDAN, H., \& TIPPER, S. P. (1998). Object-based inhibition of return in static displays. Psychonomic Bulletin \& Review, 5, 504-509.

Jordan, H., \& Tipper, S. P. (1999). Spread of inhibition across an object's surface. British Journal of Psychology, 90, 495-507.

Kahneman, D., Treisman, A., \& GibBs, B. J. (1992). The reviewing of object files: Object-specific integration of information. Cognitive Psychology, 24, 175-219.

KEPPEL, G. (1982). Design and analysis: A researcher's handbook. Englewood Cliffs, NJ: Prentice-Hall.

Kingstone, A., \& Pratt, J. (1999). Inhibition of return is composed of attentional and oculomotor processes. Perception \& Psychophysics, 61, 1046-1054.

KLEIN, R. M. (1988). Inhibitory tagging system facilitates visual search. Nature, 334, 430-431.

KleIN, R. M. (1994). Perceptual-motor expectancies interact with covert visual orienting under conditions of endogenous but not exogenous control. Canadian Journal of Experimental Psychology, 48, 167-181.

KLEIN, R. M. (2000). Inhibition of return. Trends in Cognitive Sciences, 4, 138-147.

Klein, R. M., \& MacInnes, W. J. (2000). Inhibition of return is a foraging facilitator in visual search. Psychological Science, 10, 346-352.

KLEIN, R. M., \& TAYLOR, T. L. (1994). Categories of cognitive inhibition, with reference to attention. In D. Dagenbach \& T. H. Carr (Eds.), Inhibitory processes in attention, memory, and language (pp. 113150). San Diego: Academic Press.

Kubovy, M. (1988). Should we resist the seductiveness of the space:time:: vision:audition analogy? Journal of Experimental Psychology: Human Perception \& Performance, 14, 318-320.

KWAK, H.-W., \& Egeth, H. (1992). Consequences of allocating attention to locations and to other attributes. Perception \& Psychophysics, 51, 455-464.

Lambert, A., \& Hockey, R. (1991). Peripheral visual changes and spatial attention. Acta Psychologica, 76, 149-153.

Lupiáñez, J., Milán, E. G., Tornay, F. J., Madrid, E., \& Tudela, P. (1997). Does IOR occur in discrimination tasks? Yes, it does, but later. Perception \& Psychophysics, 59, 1241-1254.

Lupiáñez, J., \& Milliken, B. (1999). Inhibition of return and the attentional set for integ rating versus differentiating information. Journal of General Psychology, 126, 392-418.

Mangun, G. R, \& Hillyard, S. A. (1991). Modulations of sensoryevoked brain potentials indicate changes in perceptual processing during visual-spatial priming. Journal of Experimental Psychology: Human Perception \& Performance, 17, 1057-1074.

MAYLOR, E. (1985). Facilitatory and inhibitory components of orienting in visual space. In M. I. Posner \& O. S. M. Marin (Eds.), Attention and performance XI (pp. 189-203). Hillsdale, NJ: Erlbaum.

McDonald, J. J., Ward, L. M., \& Kiehl, K. A. (1999). An eventrelated brain potential study of inhibition of return. Perception \& Psychophysics, 61, 1411-1423.

Milliken, B., Tipper, S. P., Houghton, G., \& Lupiáñez, J. (2000). Attending, ignoring, and repetition: On the relation between negative priming and inhibition of return. Perception \& Psychophysics, 62, 1280-1296.

Mueller, H. J., \& von Muehlenen, A. (2000). Probing distractor inhibition in visual search: Inhibition of return. Journal of Experimental Psychology: Human Perception \& Performance, 26, 1592-1605.

Oonk, H. M., \& ABrams, R. A. (1998). New perceptual objects that capture attention produce inhibition of return. Psychonomic Bulletin \& Review, 5, 510-515.

Posner, M. I. (1980). Orienting of attention. Quarterly Journal of Experimental Psychology, 32, 3-25.

Posner, M. I., \& Cohen, Y. (1984). Components of visual orienting. In H. Bouma \& D. G. Bouwhuis (Eds.), Attention and performance X: Control of language processes (pp. 531-556). Hillsdale, NJ: Erlbaum.

Posner, M. I., Rafal, R. D., Choate, L. S., \& Vaughan, J. (1985). Inhibition of return: Neural basis and function. Cognitive Neuropsychology, 2, 211-228.

Pratt, J. (1995). Inhibition of return in a discrimination task. Psychonomic Bulletin \& Review, 2, 117-120. 
Pratt, J., \& Abrams, R. A. (1999). Inhibition of return in discrimination tasks. Journal of Experimental Psychology: Human Perception \& Performance, 25, 229-242.

Pratt, J., Kingstone, A., \& Khoe, W. (1997). Inhibition of return in location- and identity-based choice decision tasks. Perception \& Psychophysics, 59, 964-971.

Rafal, R. D., Calabresi, P. A., Brennan, C. W., \& Sciolto, T. K. (1989). Saccade preparation inhibits reorienting to recently attended locations. Journal of Experimental Psychology: Human Perception \& Performance, 15, 673-685.

Rafal, R. D., Egly, R., \& Rhodes, D. (1994). Effects of inhibition of return on voluntary and visually guided saccades. Canadian Journal of Experimental Psychology, 48, 284-300.

Reuter-Lorenz, P. A., JHA, A. P., \& Rosenquist, J. N. (1996). What is inhibited in inhibition of return? Journal of Experimental Psychology: Human Perception \& Performance, 22, 367-378.

Riggio, L., Bello, A., \& UMiltà, C. (1998). Inhibitory and facilitatory effects of cue onset and offset. Psychological Research, 61, 107-118.

Ro, T., \& RAFAL, R. D. (1999). Components of reflexive visual orienting to moving objects. Perception \& Psychophysics, 61, 826-836.

Rubichi, S., Nicoletti, R, Iani, C., \& Umiltà, C. (1997). The Simon effect occurs relative to the direction of an attention shift. Journal of Experimental Psychology: Human Perception \& Performance, 23, 1353-1364.

SNydER, J. J., \& KIngSTONE, A. (2000). Inhibition of return and visual search: How many separate loci are inhibited? Perception \& Psychophysics, 62, 452-458.

TAKEDA, Y., \& YAGI, A. (2000). Inhibitory tagging in visual search can be found if search stimuli remain visible. Perception \& Psychophysics, 62, 927-934.

TANaka, T., \& Shimojo, S. (1996). Location vs. feature: Reaction time reveals dissociation between two visual functions. Vision Research, 36, 2125-2140.

Tassinari, G., Aglioti, S., Chelazzi,L., Marzi, C. A., \& Berlucchi, G. (1987). Distribution in the visual field of the costs of voluntarily allocated attention and the inhibitory after-effects of covert orienting. Neuropsychologia, 25, 55-72.

Tassinari, G., Aglioti, S., Chelazzi, L., Peru, A., \& Berlucchi, G. (1994). Do peripheral non-informative cues induce early facilitation of target detection? Vision Research, 34, 179-189.

TAssinari, G., \& Berlucchi, G. (1993). Sensory and attentional components of slowing of manual reaction time to non-fixated visual targets by ipsilateral primes. Vision Research, 11, 1525-1534.

TAY LOR, T. L. (2001). IOR following central and peripheral uninformative errors: Effects on stimulus and response repetition. Manuscript in preparation.

TAYLOR, T. L., \& KLEIN, R. M. (1998). On the causes and effects of inhibition of return. Psychonomic Bulletin \& Review, 5, 625-643.

TAYLOR, T. L., \& KLEIN, R. M. (2000). Visual and motor effects in IOR. Journal of Experimental Psychology: Human Perception \& Performance, 26, 1639-1656.

Terry, K. M., Valdes, L. A., \& Neill, W. T. (1994). Does "inhibition of return" occur in discrimination tasks? Perception \& Psychophysics, 55, 279-286.

Tipper, S. P., Driver, J., \& Weaver, B. (1991). Object-centered inhibition of return of visual attention. Quarterly Journal of Experimental Psychology, 43A, 289-298.

Tipper, S. P., Jordan, H., \& Weaver, B. (1999). Scene-based and objectcentered inhibition of return: Evidence for dual orienting mechanisms. Perception \& Psychophysics, 61, 50-60.

Tipper, S. P., Weaver, B., Jerreat, L. M., \& Burak, A. L. (1994). Object-based and environment-based inhibition of return of visual attention. Journal of Experimental Psychology: Human Perception \& Performance, 20, 478-499.

VAUGHAN, J. (1984). Saccades directed at previously attended locations in space. In A. J. Gale \& C. W. Johnson (Eds.), Theoretical and applied aspects of eye movement research (pp. 143-150). Amsterdam: North-Holland.

\section{NOTES}

1. It is not clear why these caveats hold for saccadic-manual, but not for manual-manual, response combinations. Taylor and Klein (2000) presented participants with a conceptually similar task. They required participants to determine the direction of a centrally presented arrow and make a saccade to a corresponding peripheral location. This could be followed by a second central arrow, to which participants responded with a saccade, or else with a choice manual response to indicate the direction of the arrow. Both tasks required the determination of the arrow direction and the execution of a choice response. And both tasks revealed robust IOR effects. The use of central rather than peripheral stimuli is a substantial difference from Pratt and Abrams (1999) and may account for the difference in results. Exploring this issue, however, is beyond the scope of the present investigation.

Note that we did not include the Taylor and Klein (2000) example in Table 1 or 2 . These tables summarize only studies that have required discriminations of perceptual targets. It is not clear whether the discrimination of items at fixation might operate similarly, and with respect to arrows, it is not clear whether responses made in the direction of an arrow truly reflect a visual discrimination and a choice response or whether the effects operate more exogenously (see Taylor, 2001).

2 . Because responses made in object discrimination tasks often have a spatial component, motor contributions to IOR cannot be ruled out altogether. Consider a task in which the participant is required to make a left response to a blue target and a right response to a red target, regardless of location. There is the potential for a Simon effect, wherein responses that are spatially compatible with the target are facilitated despite the fact that location is task irrelevant. For example, because blue targets require a left response, they would be responded to more quickly when presented on the left than on the right. Although there has been a suggestion that the Simon effect may be related to the locus of attention (e.g., Rubichi, Nicoletti, Iani, \& Umiltà, 1997), to the extent that it is, instead, related to responding (e.g., Buckolz, O'Donnell, \& McAuliffe, 1996), the Simon effect could potentially interact with any motor IOR effects. Indeed, in a meta-analysis of the literature, Ivanoff, Klein, and Lupiáñez (in press) demonstrated that IOR does interact with the Simon effect. However, it is worth noting that this interaction has a very small effect size and is, therefore, not typically revealed within the context of individual experiments (e.g., Lupiáñez \& Milliken, 1999); it takes a meta-analysis to reveal the effects. This does not deny the potential of motor IOR effects to impact an object discrimination, but it does suggest that any such influences are likely to be negligible within the context of a single study.

3 . To ensure that differences in computer hardware and number of trials had no effect on the results from Experiments 1 and 2, we analyzed the mean of the median S2 RT data as a function of repetition condition and participant group (Vanderbilt or Dalhousie). There were no main effects of, or interactions with, participant group for either experiment or for either discrimination condition (all $p s>.19$ ). We performed identical analyses of the mean S2 RTs and also found no main effects of, or interactions with, participant group (all $p s>.21$ ).

4. For the sake of completeness, we also analyzed the average of the mean RTs for S1 and for contextual IOR effects. The magnitude and direction of all effects were largely unchanged. The conclusions remain the same regardless of whether medians or means are used as the basis for analysis.

(Manuscript received June 30, 1999; revision accepted for publication March 22, 2001.) 